\title{
The Efficacy and Safety of Compound Danshen Dripping Pill Combined with Percutaneous Coronary Intervention for Coronary Heart Disease
}

\author{
Cailan Li, ${ }^{1}$ Qian $\mathrm{Li},{ }^{2,3}$ Jiamin $\mathrm{Xu},{ }^{4}$ Wenzhen $\mathrm{Wu},{ }^{4}$ Yuling $\mathrm{Wu},{ }^{4}$ Jianhui Xie $\mathbb{D}^{2,3}$ \\ and Xiaobo Yang $\mathbb{D}^{2,3}$ \\ ${ }^{1}$ Department of Pharmacology, Zunyi Medical University, Zhuhai Campus, Zhuhai 519041, China \\ ${ }^{2}$ State Key Laboratory of Dampness Syndrome of Chinese Medicine, \\ The Second Affiliated Hospital of Guangzhou University of Chinese Medicine, Guangzhou 510120, China \\ ${ }^{3}$ Guangdong Provincial Key Laboratory of Clinical Research on Traditional Chinese Medicine Syndrome, \\ The Second Affiliated Hospital of Guangzhou University of Chinese Medicine, Guangzhou 510120, China \\ ${ }^{4}$ The Second School of Medicine, Guangzhou University of Chinese Medicine, Guangzhou 510120, China \\ Correspondence should be addressed to Jianhui Xie; xiejianhui888@hotmail.com and Xiaobo Yang; \\ yangxiaobo39358517@hotmail.com
}

Received 30 July 2020; Revised 9 October 2020; Accepted 21 October 2020; Published 17 November 2020

Academic Editor: Mingjun Zhu

Copyright ( 92020 Cailan Li et al. This is an open access article distributed under the Creative Commons Attribution License, which permits unrestricted use, distribution, and reproduction in any medium, provided the original work is properly cited.

\begin{abstract}
Objective. Compound Danshen dripping pill (CDDP) is a well-known Chinese patent medicine, which is commonly used for the treatment of coronary heart disease (CHD) in China. This study is aimed at systematically assessing the clinical efficacy of CDDP for CHD patients. Methods. Eight databases were retrieved for eligible research studies from the founding date to April 20, 2020. Risk ratio (RR) was used to assess major adverse cardiac events (MACE) and adverse reactions, and mean difference (MD) was adopted to evaluate the hemorheology and blood lipid indexes, vascular endothelial function, cardiac function, and inflammation. Result. Twenty randomized controlled trials involving 2574 participants with CHD were included. The results indicated that, compared with percutaneous coronary intervention (PCI) alone, the combination of CDDP with PCI treatment remarkably reduced MACE $(\mathrm{RR}=0.53,95 \%$ confidence interval $(\mathrm{CI})(0.44,0.65), P<0.00001)$. Moreover, hemorheology and blood lipid parameters and inflammatory mediators of CHD patients were also dramatically mitigated after the combined therapy $(P<0.01)$. In addition, vascular endothelial function and cardiac function were prominently improved by this combination $(P<0.001)$. However, there was no significant difference in adverse reactions between the two groups $(P>0.05)$. Conclusion. Evidence from the meta-analysis demonstrated that CDDP combined with PCI treatment prominently reduced the incidence of MACE, improved cardiovascular functions, and inhibited inflammation in CHD patients. Therefore, CDDP combined with PCI treatment could be an effective and safe therapeutic method for CHD patients.
\end{abstract}

\section{Introduction}

Coronary heart disease (CHD) is one of the most serious heart diseases that threaten human health [1]. It is characterized by coronary atherosclerosis lesions aroused by myocardial ischemia, hypoxia or necrosis, stenosis, occlusion of the lumen, and inflammation [2]. The World Health
Organization (WHO) divides CHD into five categories: asymptomatic myocardial ischemia, angina pectoris, myocardial infarction, ischemic cardiomyopathy, and sudden death [3]. In recent years, in order to keep up with the continuous updating of the concept of diagnosis and treatment of $\mathrm{CHD}$ and facilitate the formulation of treatment strategies, two kinds of syndromes were proposed 
clinically, namely, chronic myocardial ischemia syndrome and acute coronary syndrome (ACS) $[4,5]$. CHD is one of the major causes of death worldwide, accounting for about one-third of all deaths [6].

The treatment of CHD mainly includes lifestyle change, drug therapy, percutaneous coronary intervention (PCI), and surgical operation [7]. At present, PCI has been the most common method for the treatment of CHD, which can effectively alleviate coronary artery stenosis or occlusion, rebuild coronary artery blood flow, and improve coronary artery blood circulation, with less trauma and obvious clinical effect [8]. However, PCI is an invasive operation, which very easily leads to vascular endothelial injury [9]. After PCI treatment, there was a high incidence of adverse cardiovascular events such as recurrent angina pectoris, coronary restenosis, acute myocardial infarction, malignant arrhythmia, and sudden death, which reduces the therapeutic effect of PCI treatment for CHD patients [10]. Therefore, how to improve the short-term efficacy and long-term prognosis of CHD patients with PCI treatment has become the direction clinical workers should strive for.

According to the basic theory of traditional Chinese medicine, the etiology and pathogenesis of CHD are associated with blood stasis. Therefore, promoting blood circulation and removing blood stasis are an important treatment for CHD. Compound Danshen dripping pill (CDDP) is a famous Chinese patent medicine approved by China Food and Drug Administration (CFDA), which has been widely used in various cardiocerebrovascular diseases $[11,12]$. CDDP is prepared from Salviae miltiorrhizae, Panax notoginseng, and Borneolum with modern techniques [13]. The main function of CDDP is promoting blood circulation to remove blood stasis and regulating qi-flowing for relieving pain [14]. In recent years, CDDP is frequently used to treat CHD sufferers combined with PCI [15]. However, most of the clinical researches could not provide sufficient evidence for the small sample sizes, and systematic evidence is lacking and urgently needed to prove the efficacy and safety. Therefore, this meta-analysis was conducted by systematically evaluating the effectiveness of CDDP combined with PCI for CHD compared with PCI therapy alone, in order to provide a scientific basis for this combination treatment.

\section{Materials and Methods}

2.1. Search Strategy. This meta-analysis was conducted according to the PRISMA statement [16]. Randomized controlled trials (RCTs) were independently searched and retrieved by two investigators (Cailan $\mathrm{Li}$ and Qian $\mathrm{Li}$ ) in the following databases from the founding date to April 20, 2020: PubMed, Embase, the Cochrane Library, Web of Science (WOS), China National Knowledge Infrastructure (CNKI), China Biology Medicine disc (CBMdisc), Wanfang data, and VIP medicine information system (VMIS). In the literature retrieval, the following search terms were used in combination: ("compound Danshen dripping pill" OR "Fufang Danshen diwan") AND ("coronary heart disease" OR "CHD" OR “coronary artery disease" OR "CAD”) AND ("percutaneous coronary intervention" OR "PCI").

2.2. Inclusion Criteria. Based on the suggestions of several specialists, the inclusion criteria were established as follows: (1) participants were diagnosed with CHD by the cardiovascular disease diagnostic criteria determined by the Chinese Medical Society (CMA) or American Heart Association (AHA) in RCTs [17, 18]; (2) all researches mentioned were described as RCTs; (3) CDDP served as the only Chinese patent medicine in RCTs; (4) sufferers in the experimental group were treated with the combined therapy of CDDP and PCI-based treatment, whereas sufferers in the control group only received PCI therapy; (5) outcome measurements of each research included at least one of the following indices: major adverse cardiac events (MACE) including recurrent angina, coronary restenosis, acute myocardial infarction, malignant arrhythmia, cardiac failure, cardiogenic shock and sudden cardiac death, hemorheology indices including whole blood viscosity (WBV), plasma viscosity (PV), hematocrit (HCT), erythrocyte aggregation index (EAI), and fibrinogen (FIB) level, vascular endothelial function indicators involving endothelin (ET), flow mediated dilation (FMD), and nitric oxide (NO), blood lipid parameters including total cholesterol (TC), triglyceride (TG), high density lipoprotein cholesterol (HDL-C), and low density lipoprotein cholesterol (LDL-C), cardiac function indicators including left ventricular ejection fraction (LVEF), left ventricular end diastolic diameter (LVEDD), and cardiac index (CI), inflammatory mediators including high-sensitivity C-reactive protein (Hs-CRP), tumor necrosis factor-alpha (TNF- $\alpha$ ), interleukin-6 (IL-6) and interleukin-8 (IL-8), and adverse reactions. Among these indices, MACE is the primary indicator, and the others belong to the secondary indicators.

2.3. Exclusion Criteria. If they demonstrated any one of the following, researches could be ruled out: (1) they were case report, editorials, and irrelevant clinical trials; (2) studies were not RCTs or diagnostic criteria were not clear; (3) the intervention of CHD patients was not conformed; (4) for the researches with data duplication, the late published study was regarded as data fraud and rejected if the authors could not be reached.

2.4. Data Extraction and Quality Evaluation. Information of eligible researches containing author names, publication year, sample size, intervention methods, outcome measurements, etc. was abstracted and is summarized in Table 1. In light of the Cochrane Handbook for Systematic Reviews of Interventions, quality assessment of included studies was independently conducted by two authors (Cailan Li and Jiamin $\mathrm{Xu}$ ) using the risk of bias table from Review Manager 5.3 [39]. There were seven kinds of biases including random sequence generation (selection bias), allocation concealment 
TABLE 1: Characteristics of the included studies.

\begin{tabular}{|c|c|c|c|c|c|c|}
\hline Study ID & $\mathrm{T} / \mathrm{C}$ & Intervention & Control & $\begin{array}{l}\text { CDDP } \\
\text { dosage }\end{array}$ & Duration & Outcome measures \\
\hline Wang et al. [19] & $15 / 33$ & CDDP + control & $\begin{array}{l}\mathrm{PCI}+\text { conventional } \\
\text { treatment }\end{array}$ & 10 pills, t.i.d. & 1 month & MACE; CF indexes \\
\hline Qiu and Zhong [20] & $32 / 30$ & $\mathrm{CDDP}+$ control & $\begin{array}{l}\mathrm{PCI}+\text { conventional } \\
\text { treatment }\end{array}$ & 10 pills, t.i.d. & 3 months & CF indexes \\
\hline Chen, 2008 [21] & $30 / 30$ & CDDP + control & $\begin{array}{l}\mathrm{PCI}+\text { conventional } \\
\text { treatment }\end{array}$ & 10 pills, t.i.d. & 3 months & $\mathrm{BL}$ and $\mathrm{CF}$ indexes \\
\hline Xuan et al. [22] & $50 / 50$ & $\mathrm{CDDP}+$ control & $\begin{array}{l}\mathrm{PCI}+\text { conventional } \\
\text { treatment }\end{array}$ & 10 pills, t.i.d. & 3 weeks & VEF and CF indexes \\
\hline Zhao et al. [23] & $83 / 77$ & $\mathrm{CDDP}+$ control & $\begin{array}{l}\mathrm{PCI}+\text { conventional } \\
\text { treatment }\end{array}$ & 10 pills, t.i.d. & 3 months & HR indexes \\
\hline Li et al. [24] & $252 / 248$ & $\mathrm{CDDP}+$ control & $\begin{array}{l}\mathrm{PCI}+\text { conventional } \\
\text { treatment }\end{array}$ & 10 pills, t.i.d. & 1 month & MACE; CF indexes \\
\hline Tian et al. [25] & $44 / 40$ & CDDP + control & $\begin{array}{l}\mathrm{PCI}+\text { conventional } \\
\text { treatment }\end{array}$ & 10 pills, t.i.d. & 3 months & MACE; BL indexes \\
\hline Xia [26] & $15 / 15$ & $\mathrm{CDDP}+$ control & $\begin{array}{l}\mathrm{PCI}+\text { conventional } \\
\text { treatment }\end{array}$ & 10 pills, t.i.d. & 2 months & BL indexes \\
\hline $\begin{array}{l}\text { Tang and Zhang } \\
\text { [27] }\end{array}$ & $44 / 44$ & $\mathrm{CDDP}+$ control & $\begin{array}{l}\mathrm{PCI}+\text { conventional } \\
\text { treatment }\end{array}$ & 10 pills, t.i.d. & 6 months & MACE; HR indexes \\
\hline Yao et al. [28] & $30 / 30$ & $\mathrm{CDDP}+$ control & $\begin{array}{l}\mathrm{PCI}+\text { conventional } \\
\text { treatment }\end{array}$ & 10 pills, t.i.d. & 3 months & HR indexes \\
\hline $\mathrm{Li}$ and Wang [29] & $42 / 42$ & CDDP + control & $\begin{array}{l}\mathrm{PCI}+\text { conventional } \\
\text { treatment }\end{array}$ & 10 pills, t.i.d. & 3 months & MACE; HR indexes \\
\hline Fang et al. [30] & $60 / 60$ & $\mathrm{CDDP}+$ control & $\begin{array}{l}\mathrm{PCI}+\text { conventional } \\
\text { treatment }\end{array}$ & 10 pills, t.i.d. & 3 months & $\mathrm{CF}$ and inflammation indexes \\
\hline Zhang [31] & $76 / 84$ & $\mathrm{CDDP}+$ control & $\begin{array}{l}\mathrm{PCI}+\text { conventional } \\
\text { treatment }\end{array}$ & 10 pills, t.i.d. & 3 months & MACE; CF indexes \\
\hline Chen et al. [32] & $60 / 60$ & $\mathrm{CDDP}+$ control & $\begin{array}{l}\mathrm{PCI}+\text { conventional } \\
\text { treatment }\end{array}$ & 10 pills, t.i.d. & 1 month & BL and inflammation indexes \\
\hline $\mathrm{Li}[33]$ & $40 / 40$ & CDDP + control & $\begin{array}{l}\mathrm{PCI}+\text { conventional } \\
\text { treatment }\end{array}$ & 10 pills, t.i.d. & 3 months & Inflammation indexes; ARs \\
\hline Tian et al. [34] & $79 / 79$ & CDDP + control & $\begin{array}{l}\mathrm{PCI}+\text { conventional } \\
\text { treatment }\end{array}$ & 10 pills, t.i.d. & 1 month & MACE; VEF indexes; ARs \\
\hline $\mathrm{Wu}$ and $\mathrm{Xu}[35]$ & $97 / 97$ & CDDP + control & $\begin{array}{l}\mathrm{PCI}+\text { conventional } \\
\text { treatment }\end{array}$ & 10 pills, t.i.d. & 1 month & $\begin{array}{c}\text { VEF and inflammation } \\
\text { indexes }\end{array}$ \\
\hline Ji et al. [36] & $67 / 69$ & CDDP + control & $\begin{array}{l}\mathrm{PCI}+\text { conventional } \\
\text { treatment }\end{array}$ & 10 pills, t.i.d. & 1 week & MACE; CF indexes \\
\hline $\mathrm{Su}[37]$ & $75 / 75$ & CDDP + control & $\begin{array}{l}\mathrm{PCI}+\text { conventional } \\
\text { treatment }\end{array}$ & 10 pills, t.i.d. & 2 months & CF indexes \\
\hline Wang et al. [38] & $90 / 90$ & $\mathrm{CDDP}+$ control & $\begin{array}{l}\mathrm{PCI}+\text { conventional } \\
\text { treatment }\end{array}$ & 10 pills, t.i.d. & 2 months & $\mathrm{CF}$ and inflammation indexes \\
\hline
\end{tabular}

T, trial group; C, control group; CDDP, compound Danshen dripping pill; PCI, percutaneous coronary intervention; t.i.d., three times a day; MACE, major adverse cardiac events; HR, hemorheology; VEF, vascular endothelial function; BL, blood lipid; CF, cardiac function; conventional treatment referred to some Western medicines mainly including nitrates, $\beta$-blockers, calcium channel blockers, statins, and platelet inhibitors.

(selection bias), blinding of participants and personnel (performance bias), blinding of outcome assessment (detection bias), incomplete outcome data (attrition bias), selective reporting (reporting bias), and other bias. Each term was judged with three grades: low risk of bias, unclear, and high risk of bias. "Low risk of bias" indicates the description of methods or procedures was adequate or correct, while "high risk of bias" represents inadequate or incorrect description. When inadequate information was shown in the study and we could not definitely judge "high risk" or "low risk," the item was judged as "unclear." Discrepancies about data abstraction and research assessment were settled by mutual discussion or consultation to a third reviewer (Jianhui Xie).
2.5. Statistical Analysis. Review Manager 5.3 (Cochrane Collaboration) was used to analyze the abstracted data from the eligible researches [39]. Outcome measures including MACE and adverse reactions were considered as dichotomous variables and presented as the risk ratio (RR) with $95 \%$ confidence interval (CI), the indices of hemorheology, vascular endothelial function, blood lipid, cardiac function, and inflammation being continuous variables that presented as the mean difference (MD) with 95\% CI. The chi-squared test was employed to check the heterogeneity among researches, and $I^{2}$ statistic was used for showing the size of heterogeneity. A fixed-effect model was adopted to analyze data with low heterogeneity $\left(P \geq 0.1\right.$ and $\left.I^{2} \leq 50 \%\right)$, and data with high heterogeneity $\left(P<0.1\right.$ or $\left.I^{2}>50 \%\right)$ was evaluated 
by a random-effect model [40]. The risk of publication bias in the included researches was revealed by a funnel plot.

\section{Results}

3.1. Study Selection. There were one hundred and twentyfive potential records from Chinese databases identified in the first review, and no related record was retrieved in English databases. Sixty-four duplicated records were removed due to the intersection of database coverage. A total of 61 records were obtained for title or abstract examination, and 22 records were dropped by reason of unrelated topics. Thirty-nine records were reserved to check full text. According to the full-text inspection, 19 studies were excluded for the following reasons: 3 researches were not RCTs, diagnosis in 7 studies was not clear, 5 trials mentioned improper interventions, and 4 articles,, respectively, showed same data with another article. In final, there were twenty researches [19-38] included in the meta-analysis (Figure 1).

3.2. Study Characteristics and Quality Assessment. Twenty eligible researches including 2574 sufferers were all published in Chinese databases from 2003 to 2019. The experimental group contained 1281 sufferers, and the control group contained 1293 sufferers. The age of all the sufferers ranged from 20 to 80 . All the included trials were RCTs with a comparison between the combined therapy of CDDP and PCI treatment and PCI treatment only. In all studies, the dose of CDDP was 10 pills each time, three times a day by oral administration. And most studies reported that the duration of treatment lasted for 3 months or so. Obvious difference was not found between the two groups in basic information (Table 1).

The methodological quality of the eligible studies was assessed by the Cochrane risk of bias evaluation and showed to be universally low. Eleven $[19,21-23,25,27-30,32,34]$ of the included trials showed the allocation sequence generation without giving the specific random method, and seven studies $[24,26,31,33,35-37]$ indicated that they were randomly grouped according to the random number table method. All the included researches did not describe allocation concealment, blinding of participants, and outcome assessment. Nine trials $[19,24,25,27,29,31,34-36]$ were at low risk of attrition bias for providing a complete outcome data. Five researches $[21,27,29,33,34]$ reporting the result of detailed indices showed a low risk of reporting bias. The risk of bias graph is shown in Figure 2.

3.3. Major Adverse cardiac events. Eight $[19,24,25,27,29,31,34,36]$ of 20 researches compared the incidence of MACE between CDDP combined with PCI treatment and single PCI treatment. A meta-analysis of the 8 studies adopting a fixed-effect model indicated that the combination therapy of CDDP and PCI treatment markedly reduced the incidence of MACE compared to single PCI treatment in treating $\mathrm{CHD}(\mathrm{RR}=0.53,95 \% \mathrm{CI}(0.44,0.65)$, $P<0.00001)$. No statistically significant heterogeneity
$\left(P=0.34, I^{2}=9 \%\right)$ was found among individual studies (Figure 3).

Furthermore, subgroup analysis was performed based on different cardiac events. There were, respectively, four $[25,27,31,34]$, three $[25,27,34]$, three $[25,29,34]$, five $[19,24,25,31,36]$, four $[24,29,31,34]$, two [24, 31], and four $[24,29,34,36]$ trials comparing the incidence of recurrent angina, coronary restenosis, acute myocardial infarction, malignant arrhythmia, cardiac failure, cardiogenic shock, and sudden cardiac death between the experimental and control groups. The results of subgroup analysis showed that CDDP could significantly reduce the incidence of recurrent angina $(\mathrm{RR}=0.20,95 \% \mathrm{CI}(0.09,0.46), P=0.0001)$, coronary restenosis $(\mathrm{RR}=0.29,95 \% \mathrm{CI}(0.12,0.72), P=0.008)$, malignant arrhythmia $(\mathrm{RR}=0.63,95 \%$ CI $(0.50,0.80)$, $P=0.0002)$, and cardiac failure $(\mathrm{RR}=0.45,95 \% \mathrm{CI}(0.24$, $0.83), P=0.01$ ), and there was no difference about the incidence of acute myocardial infarction $(\mathrm{RR}=0.42,95 \% \mathrm{CI}$ (0.14, 1.23), $P=0.11)$, cardiogenic shock $(\mathrm{RR}=1,95 \% \mathrm{CI}$ $(0.31,3.26), P=0.99)$, and sudden cardiac death $(\mathrm{RR}=0.83$, $95 \%$ CI $(0.35,1.94), P=0.66)$ between the experimental and control groups (Figure 3 ).

3.4. Hemorheology Indices. WBV, PV, HCT, EAI, and FIB were the indices of blood rheology measured in the eligible researches. There were four trials [23, 27-29] mentioned WBV (high shear). No heterogeneity was found among individual researches $\left(P=0.76, I^{2}=0 \%\right)$, so a fixed-effect model was used to conduct a meta-analysis which showed that CDDP combined with PCI treatment markedly reduced WBV (high shear) $(\mathrm{MD}=-0.4,95 \%$ CI $(-0.51,-0.29)$, $P<0.00001$; Figure 4(a). Three trials [23, 27, 29] compared WBV (middle and low shear) between the experimental and control groups. Significant heterogeneity was, respectively, found among individual researches $\left(P=0.001, I^{2}=85 \%\right.$; $\left.P=0.005, I^{2}=81 \%\right)$, and a random-effect model was adopted to carry out the meta-analysis. The pooled results showed that the combination of CDDP and PCI treatment significantly decreased WBV (middle shear) $(\mathrm{MD}=-0.86$, 95\% CI $(-1.31,-0.41), P=0.0002$; Figure $4(\mathrm{~b}))$ and WBV (low shear) $(\mathrm{MD}=-0.87,95 \% \mathrm{CI}(-1.46,-0.27), P=0.0004$; Figure 4(c)).

There were, respectively, four studies [23, 27-29] that reported PV and two studies $[23,27]$ that reported HCT and EAI. No heterogeneity was, respectively, found among individual researches $\left(P=0.87, I^{2}=0 \% ; P=0.84, I^{2}=0 \%\right.$; $\left.P=0.48, I^{2}=0 \%\right)$, and a fixed-effect model was adopted to carry out the meta-analysis. The pooled results showed that the combination of CDDP and PCI treatment significantly decreased PV $(\mathrm{MD}=-0.26,95 \%$ CI $\quad(-0.3,-0.21)$, $P<0.00001$; Figure $4(\mathrm{~d}))$ and EAI $(\mathrm{MD}=-0.41,95 \% \mathrm{CI}$ $(-0.55,-0.28), P<0.00001$; Figure $4(\mathrm{f}))$, and there was no difference about HCT between the experimental and control groups $(\mathrm{MD}=0.67,95 \%$ CI $(-0.98,2.33), \quad P=0.43$; Figure 4(e)).

Three studies $[23,27,29]$ reported the level of FIB in blood plasma. Significant heterogeneity was observed among individual researches $\left(P<0.0001, I^{2}=90 \%\right)$ and then a 


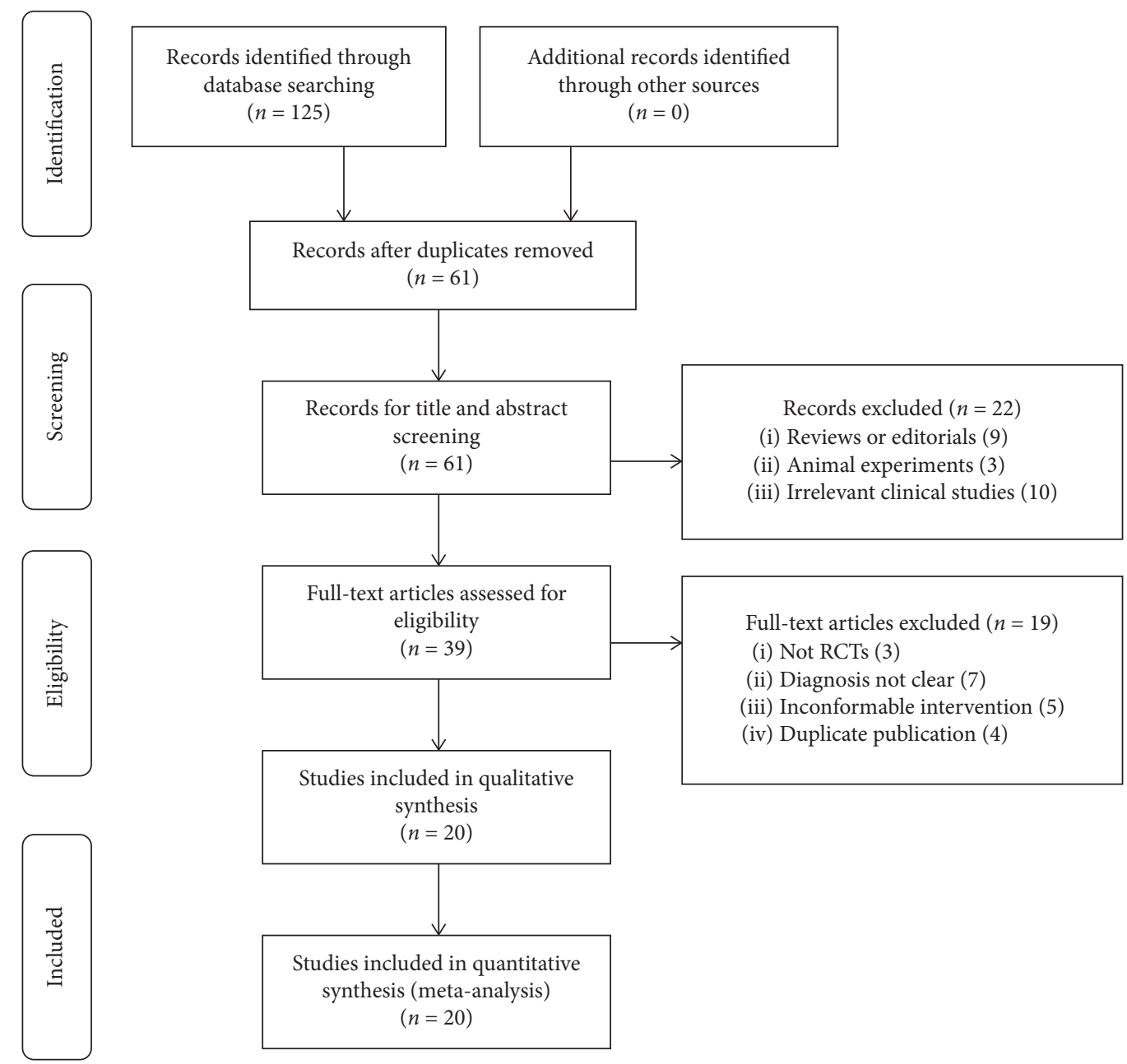

FIGURE 1: Flow diagram of study searching and screening for the meta-analysis.

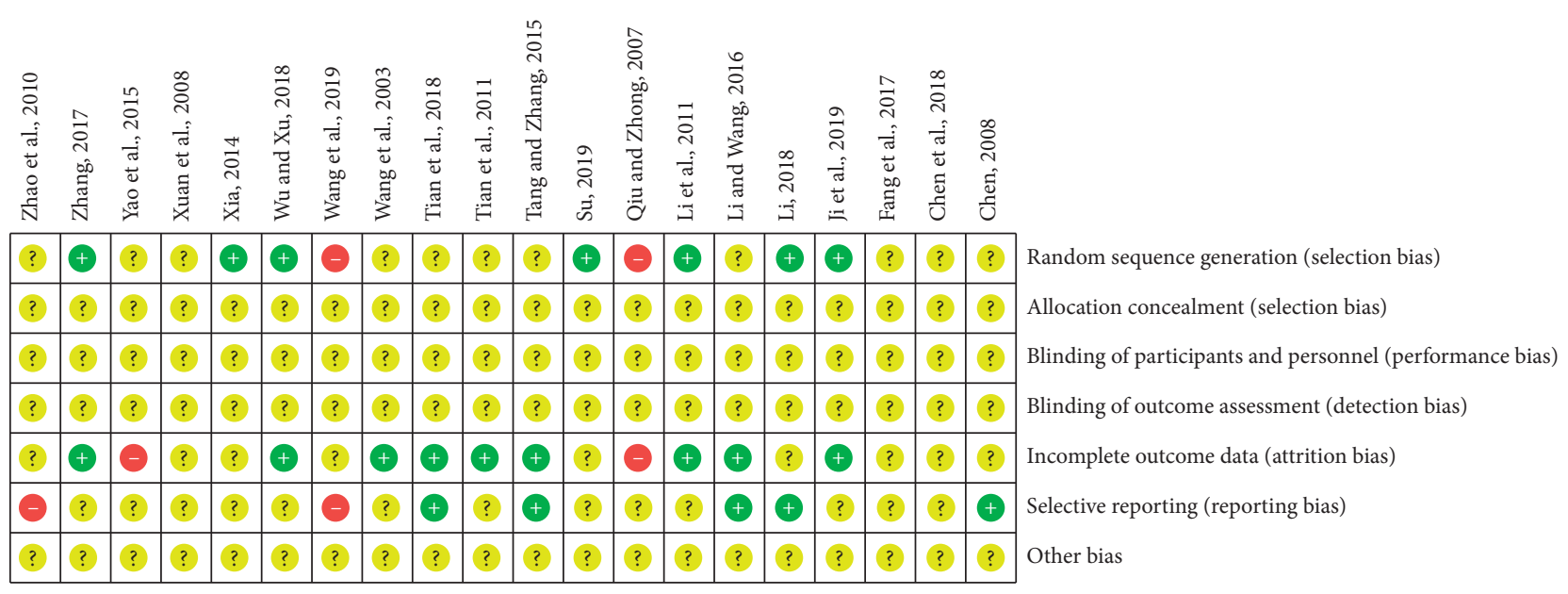

FIGURE 2: Methodological quality assessment for the risk of bias in the included studies.

random-effect meta-analysis was conducted to indicate that there was no difference about FIB between the experimental and control groups $(\mathrm{MD}=0.22,95 \%$ CI $(-0.75,1.19)$, $P=0.66$; Figure $4(\mathrm{~g}))$.
3.5. Vascular Endothelial Function Indices. ET, FMD, and NO were the indices of vascular endothelial function measured in the included studies. There were, respectively, three $[22,34,35]$, two $[22,35]$, and two $[22,34]$ studies 


\begin{tabular}{|c|c|c|c|c|c|c|c|c|c|}
\hline \multirow{2}{*}{ Study or subgroup } & \multicolumn{2}{|c|}{ Experimental } & \multicolumn{2}{|c|}{ Control } & \multirow{2}{*}{$\begin{array}{c}\text { Weight } \\
(\%)\end{array}$} & \multirow{2}{*}{$\begin{array}{l}\text { Risk ratio } \\
\text { M-H, fixed, 95\% CI }\end{array}$} & \multirow{2}{*}{\multicolumn{3}{|c|}{$\begin{array}{c}\text { Risk ratio } \\
\text { M-H, fixed, 95\% CI }\end{array}$}} \\
\hline & Events & Total & Events & Total & & & & & \\
\hline \multicolumn{10}{|l|}{ 1.1.1. Recurrent angina } \\
\hline Tian et al., 2011 & 0 & 44 & 9 & 40 & 4.2 & $0.05[0.00,0.80]$ & & & \\
\hline Tang and Zhang, 2015 & 2 & 44 & 9 & 44 & 3.8 & $0.22[0.05,0.97]$ & & & \\
\hline Zhang, 2017 & 2 & 76 & 9 & 84 & 3.6 & $0.25[0.05,1.10]$ & & & \\
\hline Tian et al., 2018 & 2 & 79 & 5 & 79 & 2.1 & $0.40[0.08,2.00]$ & & 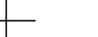 & \\
\hline Subtotal (95\% CI) & & 243 & & 247 & 13.6 & $0.20[0.09,0.46]$ & & & \\
\hline Total events & 6 & & 32 & & & & & & \\
\hline \multicolumn{10}{|c|}{$\begin{array}{l}\text { Heterogeneity: chi }{ }^{2}=1.77, \mathrm{~d} f=3(P=0.62) ; I^{2}=0 \% \\
\text { Test for overall effect: } Z=3.83(P=0.0001)\end{array}$} \\
\hline \multicolumn{10}{|l|}{ 1.1.2. Coronary restenosis } \\
\hline Tian et al., 2011 & 0 & 44 & 4 & 40 & 2.0 & $0.10[0.01,1.82]$ & & - & \\
\hline Tang and Zhang, 2015 & 4 & 44 & 12 & 44 & 5.0 & $0.33[0.12,0.95]$ & & & \\
\hline Tian et al., 2018 & 1 & 79 & 2 & 79 & 0.8 & $0.50[0.05,5.40]$ & & & \\
\hline Subtotal (95\% CI) & & 167 & & 163 & 7.8 & $0.29[0.12,0.72]$ & & & \\
\hline Total events & 5 & & 18 & & & & & & \\
\hline \multicolumn{10}{|c|}{ Heterogeneity: chi $^{2}=0.77, \mathrm{~d} f=2(P=0.68) ; I^{2}=0 \%$} \\
\hline \multicolumn{10}{|c|}{ 1.1.3. Acute myocardial infarction } \\
\hline Tian et al., 2011 & 0 & 44 & 3 & 40 & 1.5 & $0.13[0.01,2.44]$ & & & \\
\hline Li and Wang, 2016 & 3 & 42 & 4 & 42 & 1.7 & $0.75[0.18,3.15]$ & & & \\
\hline Tian et al., 2018 & 1 & 79 & 3 & 79 & 1.3 & $0.33[0.04,3.14]$ & & & \\
\hline Subtotal (95\% CI) & & 165 & & 161 & 4.5 & $0.42[0.14,1.23]$ & & & \\
\hline Total events & 4 & & 10 & & & & & & \\
\hline \multicolumn{10}{|c|}{ Heterogeneity: $\mathrm{chi}^{2}=1.28, \mathrm{~d} f=2(P=0.53) ; I^{2}=0 \%$} \\
\hline 1.1.4. Malignant arrhyth & & & & & & & & & \\
\hline Wang et al,. 2003 & 8 & 15 & 15 & 33 & 3.9 & $1.17[0.64,2.14]$ & & - & \\
\hline Li et al., 2011 & 45 & 252 & 79 & 248 & 33.3 & $0.56[0.41,0.77]$ & - & & \\
\hline Tian et al., 2011 & 0 & 44 & 1 & 40 & 0.7 & $0.30[0.01,7.25]$ & & & \\
\hline Zhang, 2017 & 5 & 76 & 15 & 84 & 6.0 & $0.37[0.14,0.97]$ & & & \\
\hline Ji et al., 2019 & 21 & 67 & 26 & 69 & 10.7 & $0.83[0.52,1.33]$ & & & \\
\hline Subtotal (95\% CI) & & 454 & & 474 & 54.5 & $0.63[0.50,0.80]$ & & & \\
\hline Total events & 79 & & 136 & & & & & & \\
\hline $\begin{array}{l}\text { Heterogeneity: chi }{ }^{2}=7 \\
\text { Test for overall effect: } Z\end{array}$ & $\begin{array}{l}0, \mathrm{~d} f=4( \\
3.79(P=\end{array}$ & $\begin{array}{l}P=0.12 \\
=0.0002\end{array}$ & $I^{2}=4$ & & & & & & \\
\hline 1.1.5. Cardiac failure & & & & & & & & & \\
\hline Li et al ., 2011 & 6 & 252 & 8 & 248 & 3.4 & $0.74[0.26,2.10]$ & & - & \\
\hline Li and Wang, 2016 & 6 & 42 & 13 & 42 & 5.4 & $0.46[0.19,1.10]$ & & & \\
\hline Zhang, 2017 & 1 & 76 & 8 & 84 & 3.2 & $0.14[0.02,1.08]$ & & & \\
\hline Tian et al., 2018 & 0 & 79 & 1 & 79 & 0.6 & $0.33[0.01,8.06]$ & & & \\
\hline Subtotal (95\% CI) & & 449 & & 453 & 12.6 & $0.45[0.24,0.83]$ & & & \\
\hline Total events & 13 & & 30 & & & & & & \\
\hline Heterogeneity: $\mathrm{chi}^{2}=2$. & $8, \mathrm{~d} f=3($ & $P=0.54$ & 4); $I^{2}=0$ & & & & & & \\
\hline Test for overall effect: $Z$ & $2.58(P=$ & $=0.010)$ & & & & & & & \\
\hline 1.1.6. Cardiogenic shock & & & & & & & & & \\
\hline Li et al ., 2011 & 5 & 252 & 4 & 248 & 1.7 & $1.23[0.33,4.53]$ & & & \\
\hline Zhang, 2017 & 0 & 76 & 1 & 84 & 0.6 & $0.37[0.02,8.90]$ & & & \\
\hline Subtotal (95\% CI) & & 328 & & 332 & 2.3 & $1.00[0.31,3.26]$ & & & \\
\hline Total events & 5 & & 5 & & & & & & \\
\hline Heterogeneity: $\mathrm{chi}^{2}=0$. & $7, \mathrm{~d} f=1($ & $P=0.49$ & 9); $I^{2}=0$ & & & & & & \\
\hline Test for overall effect: $Z$ & $0.01(P=$ & $=0.99)$ & & & & & & & \\
\hline 1.1.7. Sudden cardiac de & & & & & & & & & \\
\hline Li et al ., 2011 & 6 & 252 & 4 & 248 & 1.7 & $1.48[0.42,5.17]$ & & & \\
\hline Li and Wang, 2016 & 1 & 42 & 1 & 42 & 0.4 & $1.00[0.06,15.47]$ & & & \\
\hline Tian et al., 2018 & 0 & 79 & 1 & 79 & 0.6 & $0.33[0.01,8.06]$ & & & \\
\hline Ji et al., 2019 & 2 & 67 & 5 & 69 & 2.1 & $0.41[0.08,2.05]$ & & & \\
\hline Subtotal (95\% CI) & & 440 & & 438 & 4.8 & $0.83[0.35,1.94]$ & & & \\
\hline Total events & 9 & & 11 & & & & & & \\
\hline Heterogeneity: chi $^{2}=1$. & $8, \mathrm{~d} f=3$ & $P=0.60$ & )$; I^{2}=0$ & & & & & & \\
\hline Test for overall effect: $Z$ & $0.44(P=$ & $=0.66)$ & & & & & & & \\
\hline Total events & 121 & & 242 & & & & $\nabla$ & & \\
\hline Total (95\% CI) & & 2246 & & 2268 & 100 & $0.53[0.44,0.65]$ & & & \\
\hline Heterogeneity: chi $^{2}=26$ & $24, \mathrm{~d} f=2$ & $4(P=0$ & $34) ; I^{2}=$ & $=9 \%$ & & & & & \\
\hline Test for overall effect: $Z$ & $6.34(P$ & 0.0000 & & & & 100 & 0.005 & 1 & 200 \\
\hline Test for subgroup differ & aces: chi $^{2}$ & $=11.55$ & $\mathrm{~d} f=6$ & $(P=0.0$ & $; I^{2}=4$ & $48.0 \%$ & Favours (experimental) & Favol & ours (control) \\
\hline
\end{tabular}

FIgURe 3: Forest plot of major adverse cardiac events of CDDP plus PCI treatment compared to PCI treatment alone for CHD patients. $I^{2}$ and $P$ are the criterion for the heterogeneity test, $\bullet$ pooled risk ratio, $-\mathbf{-}-$ risk ratio, and $95 \%$ CI.

reporting ET, FMD, and NO. Significant heterogeneity was, respectively, found among individual studies $(P<0.00001$, $\left.I^{2}=99 \% ; P<0.00001, I^{2}=99 \% ; P=0.001, I^{2}=90 \%\right)$, and a random-effect model was adopted to carry out the metaanalysis. The pooled results showed that the combination of CDDP and PCI treatment significantly decreased ET 


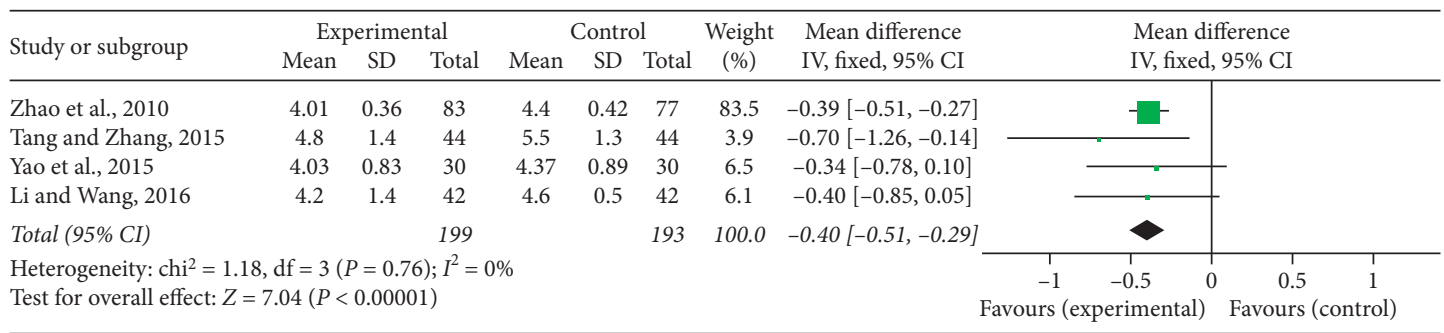

(a)

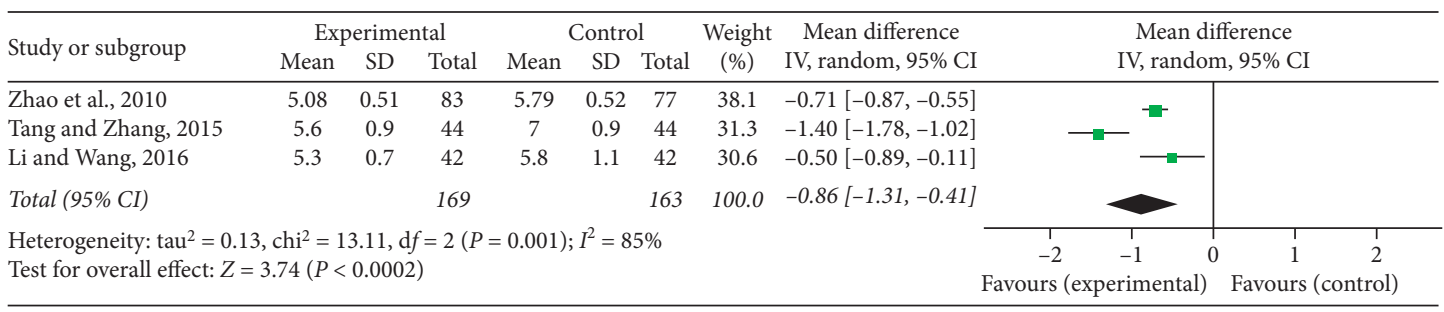

(b)

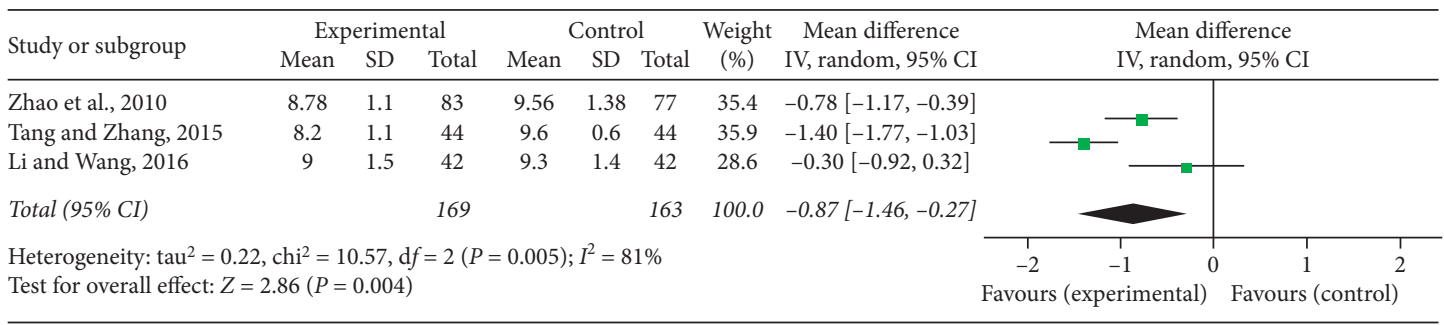

(c)

\begin{tabular}{|c|c|c|c|c|c|c|c|c|c|c|c|}
\hline \multirow{3}{*}{$\frac{\text { Study or subgroup }}{\text { Zhao et al., } 2010}$} & \multicolumn{3}{|c|}{ Experimental } & \multicolumn{3}{|c|}{ Control } & \multirow{2}{*}{$\begin{array}{l}\text { Weight } \\
(\%)\end{array}$} & \multirow{2}{*}{$\begin{array}{l}\text { Mean difference } \\
\text { IV, fixed, } 95 \% \text { CI }\end{array}$} & \multirow{2}{*}{\multicolumn{3}{|c|}{$\begin{array}{l}\text { Mean difference } \\
\text { IV, fixed, } 95 \% \text { CI }\end{array}$}} \\
\hline & Mean & SD & Total & Mean & SD & Total & & & & & \\
\hline & 1.22 & 0.12 & 83 & 1.48 & 0.16 & 77 & 89.7 & $-0.26[-0.30,-0.22]$ & & & \\
\hline Tang and Zhang, 2015 & 1.7 & 0.9 & 44 & 2 & 0.7 & 44 & 1.5 & $-0.30[-0.64,0.04]$ & & & \\
\hline Yao et al., 2015 & 1.14 & 0.48 & 30 & 1.34 & 0.26 & 30 & 4.6 & $-0.20[-0.40,-0.00]$ & & & \\
\hline Li and Wang, 2016 & 1.2 & 0.6 & 42 & 1.4 & 0.3 & 42 & 4.2 & $-0.20[-0.40,-0.00]$ & & & \\
\hline \multicolumn{3}{|l|}{ Total (95\% CI) } & 199 & & & 193 & 100.0 & $-0.26[-0.30,-0.21]$ & & & \\
\hline \multirow{2}{*}{\multicolumn{9}{|c|}{$\begin{array}{l}\text { Heterogeneity: } \text { chi }^{2}=0.70, \mathrm{~d} f=3(P=0.87) ; I^{2}=0 \% \\
\text { Test for overall effect: } Z=11.99(P<0.00001)\end{array}$}} & -0.5 & -0.25 & 0.25 \\
\hline & & & & & & & & & Favours (e & xperimental) & Favours (control) \\
\hline
\end{tabular}

(d)

\begin{tabular}{|c|c|c|c|c|c|c|c|c|c|}
\hline \multirow{2}{*}{ Study or subgroup } & \multicolumn{3}{|c|}{ Experimental } & \multicolumn{3}{|c|}{ Control } & \multirow{2}{*}{$\begin{array}{l}\text { Weight } \\
(\%)\end{array}$} & \multirow{2}{*}{$\begin{array}{l}\text { Mean difference } \\
\text { IV, fixed, 95\% CI }\end{array}$} & \multirow{2}{*}{$\begin{array}{l}\text { Mean difference } \\
\text { IV, fixed, 95\% CI }\end{array}$} \\
\hline & Mean & $\mathrm{SD}$ & Total & Mean & SD & Total & & & \\
\hline Zhao et al., 2010 & 44.96 & 6.62 & 83 & 44.24 & 4.26 & 77 & 93.7 & $0.72[-0.99,2.43]$ & \\
\hline Tang and Zhang, 2015 & 50 & 10 & 44 & 50 & 20 & 44 & 6.3 & $0.00[-6.61,6.61]$ & -1 \\
\hline Total (95\% CI) & & & 127 & & & 121 & 100.0 & $0.67[-0.98,2.33]$ & \\
\hline $\begin{array}{l}\text { Heterogeneity: } \text { chi }^{2}=0 \\
\text { Test for overall effect: } Z\end{array}$ & $\begin{array}{l}\mathrm{df}=1( \\
.80(P=\end{array}$ & $\begin{array}{l}=0.8 \\
0.43)\end{array}$ & $; I^{2}=$ & & & & & -10 & \begin{tabular}{c|c} 
& 1 \\
-5 & 0 \\
Favours (control) & Favours
\end{tabular} \\
\hline
\end{tabular}

(e)

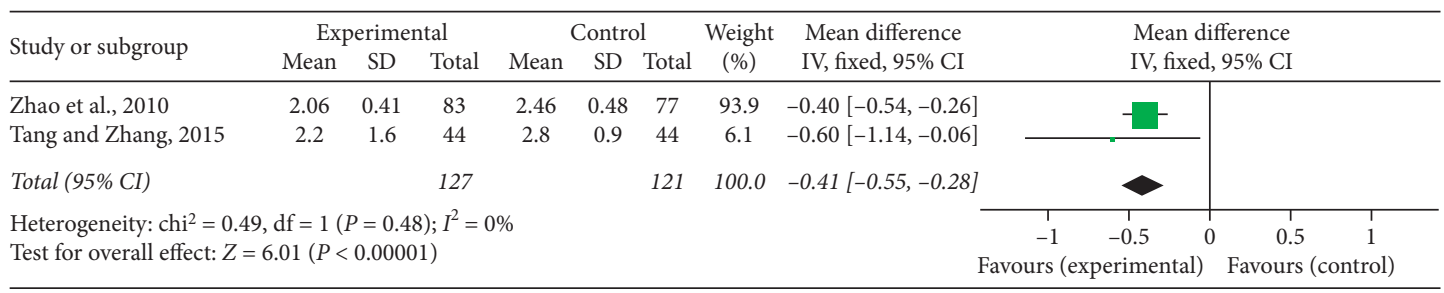

(f)

Figure 4: Continued. 


\begin{tabular}{|c|c|c|c|c|c|c|c|c|c|c|c|}
\hline \multirow{3}{*}{$\begin{array}{l}\text { Study or subgroup } \\
\text { Tang and Zhang, } 2015\end{array}$} & \multicolumn{3}{|c|}{ Experimental } & \multicolumn{3}{|c|}{ Control } & \multirow{2}{*}{$\begin{array}{l}\text { Weight } \\
(\%)\end{array}$} & \multirow{2}{*}{$\begin{array}{c}\text { Mean difference } \\
\text { IV, random, 95\% CI }\end{array}$} & \multirow{2}{*}{\multicolumn{3}{|c|}{$\begin{array}{c}\text { Mean difference } \\
\text { IV, random, 95\% CI }\end{array}$}} \\
\hline & \multirow{2}{*}{$\frac{\text { Mean }}{4.3}$} & \multirow{2}{*}{$\frac{\mathrm{SD}}{0.6}$} & \multirow{2}{*}{$\frac{\text { Total }}{44}$} & \multirow{2}{*}{$\frac{\text { Mean }}{3.3}$} & \multirow{2}{*}{$\begin{array}{l}\mathrm{SD} \\
0.8\end{array}$} & \multirow{2}{*}{$\frac{\text { Total }}{44}$} & & & & & \\
\hline & & & & & & & 36.5 & $1.00[0.70,1.30]$ & & & \\
\hline Yao et al., 2015 & 3.26 & 1.72 & 30 & 3.41 & 1.38 & 30 & 30.2 & -0.15 [ & & - & \\
\hline Li and Wang, 2016 & 3.2 & 1.5 & 42 & 3.5 & 1.2 & 42 & 33.3 & $-0.30[-0.88$, & & - & \\
\hline Total (95\% CI) & & & 116 & & & 116 & 100.0 & $0.22[-0.75,1.19]$ & & & \\
\hline $\begin{array}{l}\text { Heterogeneity: } \operatorname{tau}^{2}= \\
\text { Test for overall effect: }\end{array}$ & $\begin{array}{l}\operatorname{chi}^{2}=1 \\
.45(P=\end{array}$ & $\begin{array}{l}9.61, \mathrm{~d} \\
0.66)\end{array}$ & $=2(1$ & 0.000 & $2-c$ & $90 \%$ & & & $\begin{array}{lc} & 1 \\
-2 & -1 \\
\text { Favours (control) }\end{array}$ & $\begin{array}{ll}0 & 1 \\
\text { Favours (ex }\end{array}$ & $\begin{array}{c}1 \\
2 \\
\text { menta }\end{array}$ \\
\hline
\end{tabular}

(g)

FIgURe 4: Forest plot of comparison in two groups for hemorheology indices.(a)-(c) Whole blood viscosity (high, middle, and low shear); (d) plasma viscosity; (e) hematocrit; (f) erythrocyte aggregation index; (g) fibrinogen level. $I^{2}$ and $P$ are the criterion for the heterogeneity test, $\bullet$ pooled mean difference, $-\boldsymbol{\square}-$ mean difference, and 95\% CI.

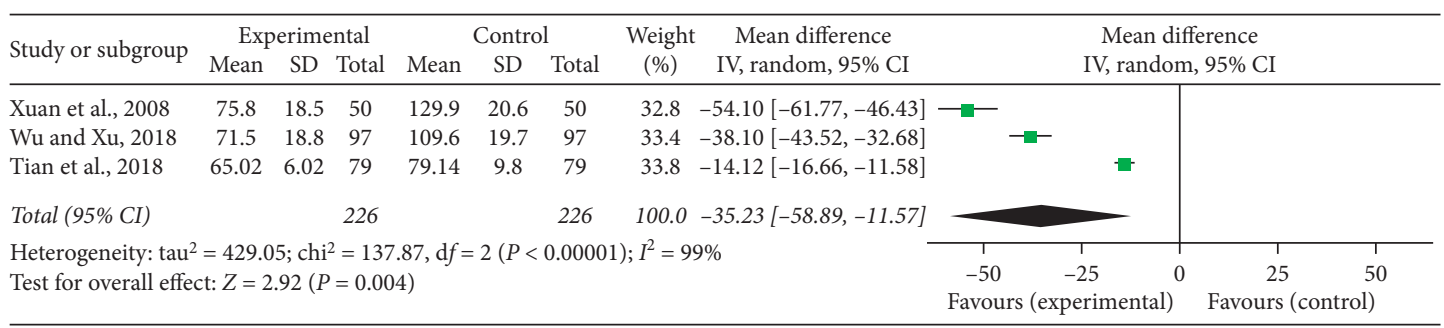

(a)

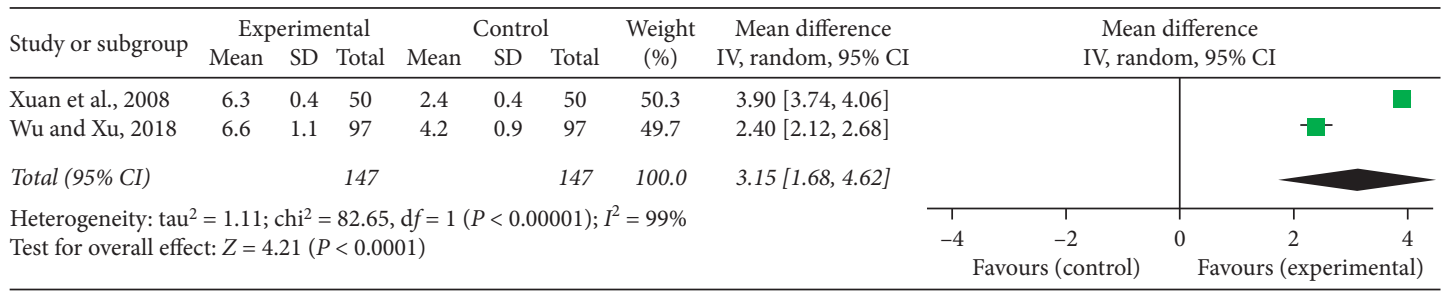

(b)

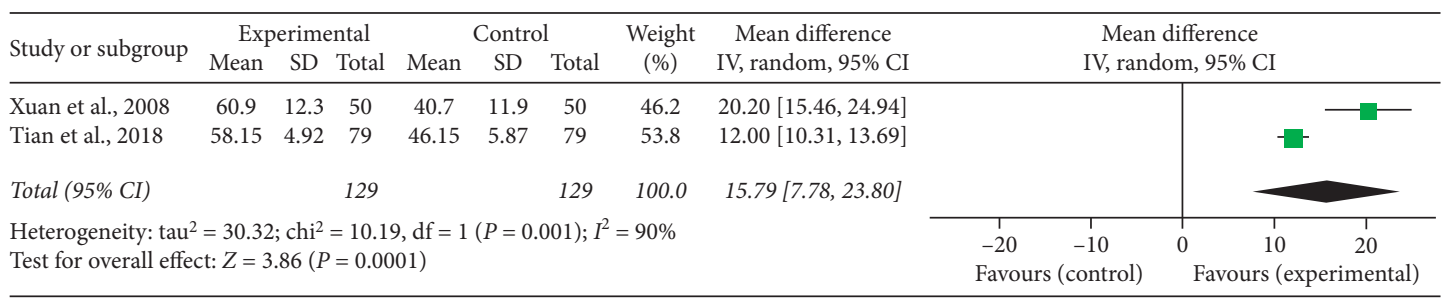

(c)

FIGURE 5: Forest plot of comparison in two groups for vascular endothelial function indices. (a) Endothelin; (b) flow mediated dilation; (c) nitric oxide. $I^{2}$ and $P$ are the criterion for the heterogeneity test, $\bullet$ pooled mean difference, $-\boldsymbol{\square}-$ mean difference, and $95 \%$ CI.

$(\mathrm{MD}=-35.23, \quad 95 \% \quad$ CI $\quad(-58.89, \quad-11.57), \quad P=0.004$ Figure $5(\mathrm{a}))$ and improved FMD $(\mathrm{MD}=3.15,95 \% \mathrm{CI}(1.68$, 4.62), $P<0.0001$; Figure 5(b)) and NO $(\mathrm{MD}=15.79,95 \% \mathrm{CI}$ (7.78, 23.8), $P=0.0001$; Figure 5(c)).

3.6. Blood Lipid Indices. TC, TG, HDL-C, and LDL-C were the indices of blood lipid measured in the included studies. There were four studies $[21,25,26,32]$ that reported TC, TG, HDL-C, and LDL-C. Significant heterogeneity was, respectively, found among individual researches $(P<0.00001$, $I^{2}=88 \% ; \quad P<0.00001, \quad I^{2}=95 \% ; \quad P<0.00001, \quad I^{2}=87 \%$; $P<0.00001, I^{2}=88 \%$ ), and a random-effect model was adopted to carry out the meta-analysis. The pooled results showed that CDDP combined with PCI treatment significantly decreased TC $(\mathrm{MD}=-0.32,95 \% \mathrm{CI}(-0.53,-0.11)$, $P=0.003$; Figure $6(\mathrm{a}))$ and LDL-C $(\mathrm{MD}=-0.38,95 \% \mathrm{CI}$ $(-0.59,-0.18), P=0.0002$; Figure $6(\mathrm{~d}))$, and there was no difference about TG (MD $=-0.23,95 \%$ CI $(-0.48,0.02)$, $P=0.07$; Figure $6(\mathrm{~b}))$ and HDL-C $(\mathrm{MD}=0.15,95 \% \mathrm{CI}$ $(-0.03,0.33), P=0.11$; Figure $6(\mathrm{c}))$ between the experimental and control groups.

3.7. Cardiac Function Indices. LVEF, LVEDD, and CI were the indices of cardiac function measured in the included studies. There were nine $[19-22,24,31,36-38]$ and five $[20,22,24,30,36]$ studies which reported LVEF and 


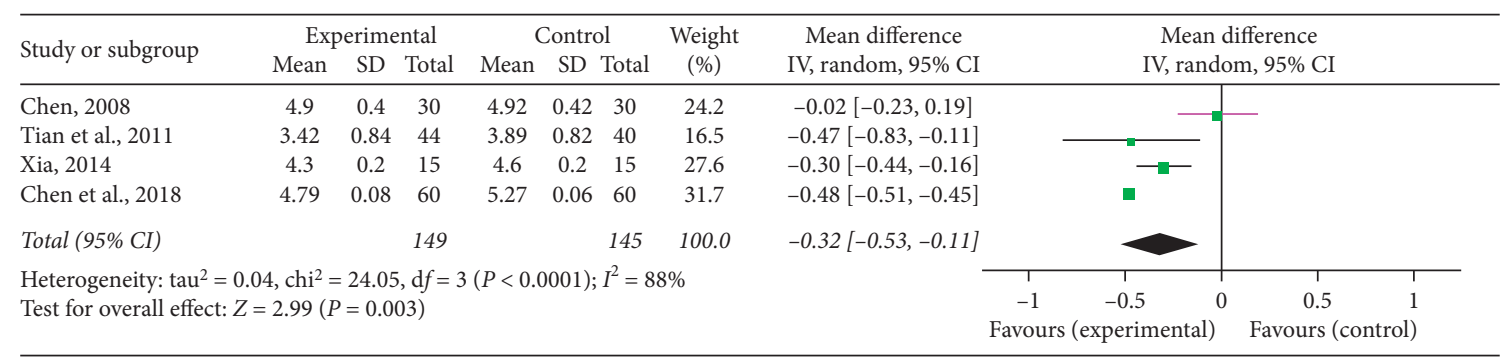

(a)

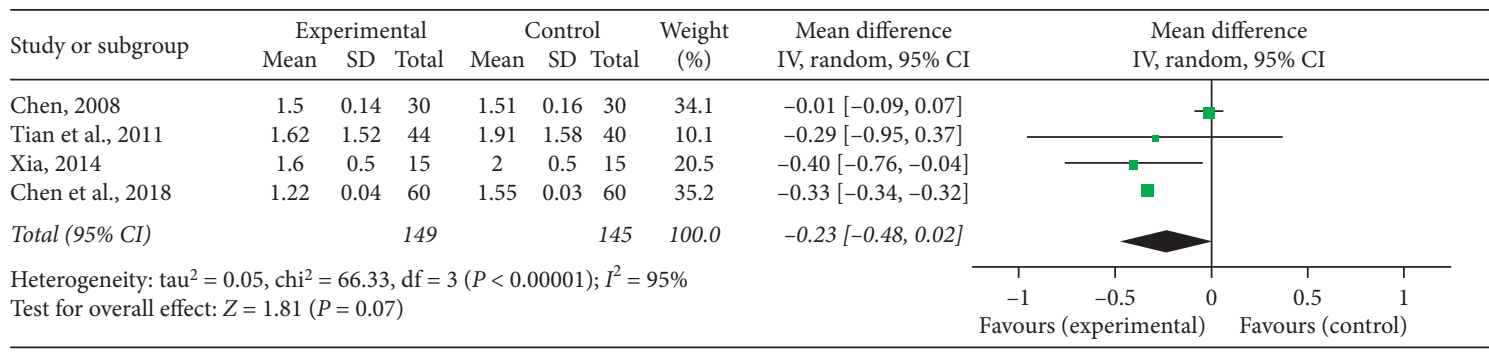

(b)

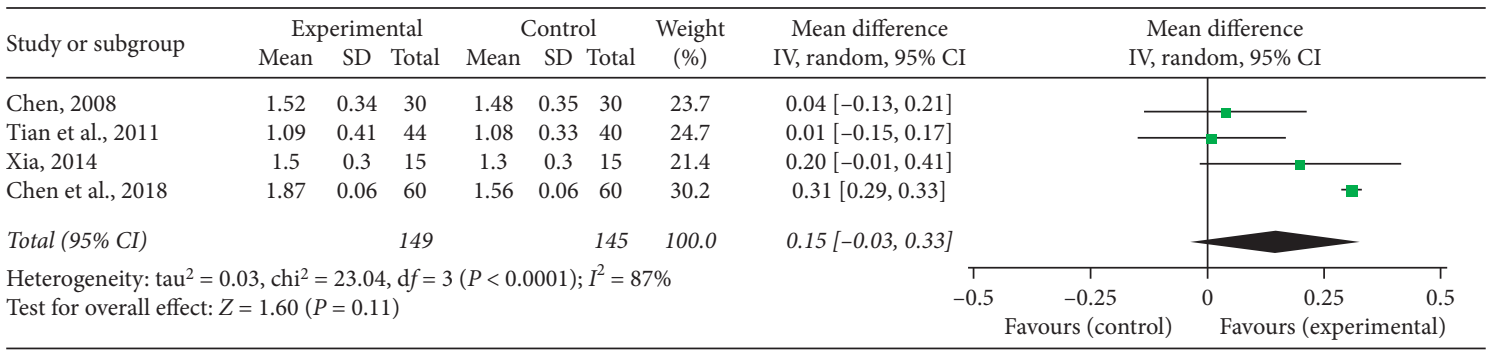

(c)

\begin{tabular}{|c|c|c|c|c|c|c|c|c|c|c|}
\hline \multirow{3}{*}{$\begin{array}{l}\text { Study or subgroup } \\
\text { Chen, } 2008\end{array}$} & \multicolumn{3}{|c|}{ Experimental } & \multicolumn{3}{|c|}{ Control } & \multirow{3}{*}{$\begin{array}{c}\begin{array}{c}\text { Weight } \\
(\%)\end{array} \\
19.5\end{array}$} & \multirow{3}{*}{$\begin{array}{c}\text { Mean difference } \\
\text { IV, random, 95\% CI } \\
-0.49[-0.77,-0.21]\end{array}$} & \multirow{2}{*}{\multicolumn{2}{|c|}{$\begin{array}{c}\text { Mean difference } \\
\text { IV, random, 95\% CI }\end{array}$}} \\
\hline & \multirow{2}{*}{$\frac{\text { Mean }}{2.32}$} & \multirow{2}{*}{$\frac{S D}{0.53}$} & \multirow{2}{*}{$\frac{\text { Total }}{30}$} & \multirow{2}{*}{$\frac{\text { Mean }}{2.81}$} & \multirow{2}{*}{$\frac{\mathrm{SD} 7}{0.58}$} & \multirow{2}{*}{$\frac{\text { Total }}{30}$} & & & & \\
\hline & & & & & & & & & - & \\
\hline Tian et al., 2011 & 1.6 & 0.59 & 44 & 2.12 & 0.46 & 40 & 22.5 & $-0.52[-0.75,-0.29]$ & & \\
\hline Xia, 2014 & 2.3 & 0.2 & 15 & 2.4 & 0.2 & 15 & 27.0 & $-0.10[-0.24,0.04]$ & . & \\
\hline Chen et al., 2018 & 2.47 & 0.05 & 60 & 2.93 & 0.07 & 60 & 31.0 & $-0.46[-0.48,-0.44]$ & $=$ & \\
\hline Total (95\% CI) & & & 149 & & & 145 & 100.0 & $-0.38[-0.59,-0.18]$ & & \\
\hline $\begin{array}{l}\text { Heterogeneity: } \operatorname{tau}^{2} \\
\text { Test for overall effec }\end{array}$ & $\begin{array}{l}3, \mathrm{chi}^{2}= \\
=3.67(P\end{array}$ & $\begin{array}{l}24.17 \\
=0.0\end{array}$ & $\begin{array}{l}\mathrm{d} f=3 \\
002)\end{array}$ & $(P<0.0$ & $0001)$ & $I^{2}=\varepsilon$ & & & $\begin{array}{c}-0.5-0.25 \\
\text { Favours (experimental) }\end{array}$ & $\begin{array}{cc} & 1 \\
0.25 & 0.5 \\
\text { Favours } & \text { (control) }\end{array}$ \\
\hline
\end{tabular}

(d)

FIGURE 6: Forest plot of comparison in two groups for blood lipid indices. (a) Total cholesterol; (b) triglyceride; (c) high density lipoprotein cholesterol; (d) low density lipoprotein cholesterol. $I^{2}$ and $P$ are the criterion for the heterogeneity test, $\bullet$ pooled mean difference, $-\boldsymbol{-}-$ mean difference, and 95\% CI.

LVEDD. Significant heterogeneity was, respectively, found among individual studies $\left(P=0.02, I^{2}=57 \% ; P<0.0001\right.$, $I^{2}=85 \%$ ), and a random-effect model was adopted to carry out the meta-analysis. The pooled results showed that CDDP combined with PCI treatment significantly improved LVEF $(\mathrm{MD}=3.46,95 \%$ CI $(2.15,4.77), P<0.0001$; Figure $7(\mathrm{a}))$ and decreased LVEDD $(\mathrm{MD}=-2.5,95 \% \mathrm{CI}$ (-3.93, -1.08), $P=0.0006$; Figure 7(b)). Two studies $[20,38]$ recorded the detection of CI. There was no heterogeneity $\left(P=0.86, I^{2}=0 \%\right)$ and a fixed-effect model was adopted to carry out the meta-analysis. The pooled result showed that the combination therapy of CDDP and PCI treatment significantly improved CI compared to single
PCI treatment $(\mathrm{MD}=1.11,95 \% \mathrm{CI}(0.8,1.43), P<0.00001$; Figure $7(\mathrm{c}))$.

3.8. Inflammatory Mediators Production. Hs-CRP, TNF- $\alpha$, IL-6, and IL- 8 were the indices of inflammation measured in the included studies. Two studies [21, 33] mentioned the investigation on Hs-CRP. No statistically significant heterogeneity $\left(P=0.34, I^{2}=0 \%\right)$ was detected in the metaanalysis and a fixed-effect model was used. An OR with $95 \%$ CI was adopted to present the comparison of Hs-CRP between the experimental and control groups $(\mathrm{MD}=-0.74$, 95\% CI $(-1.05,-0.42), P<0.00001$; Figure $8(\mathrm{a}))$. It indicated 


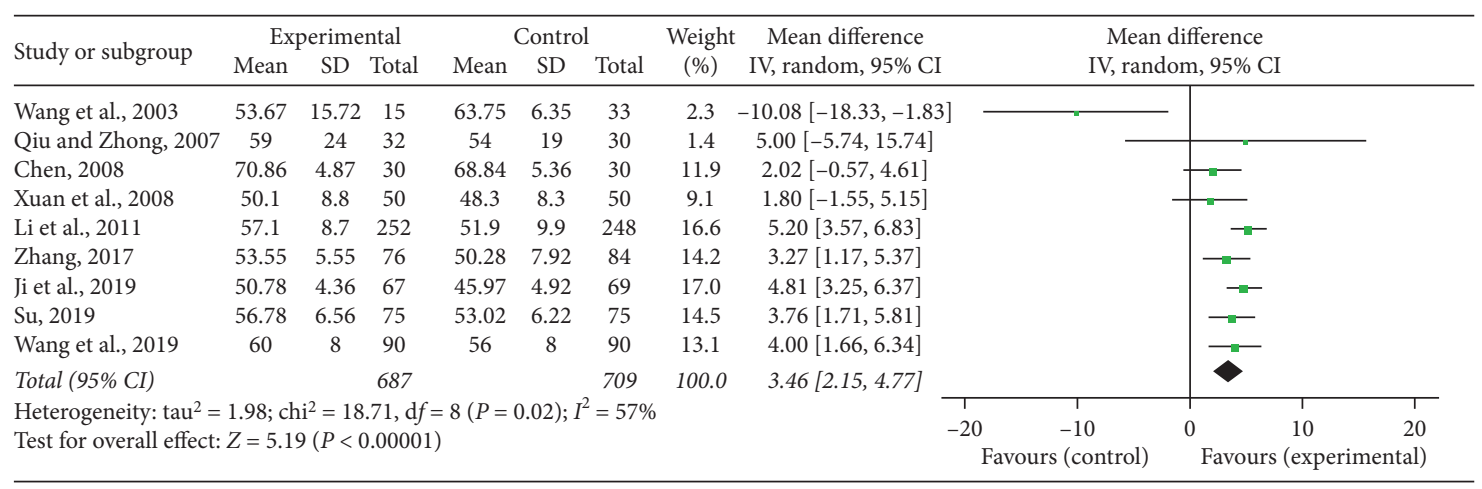

(a)

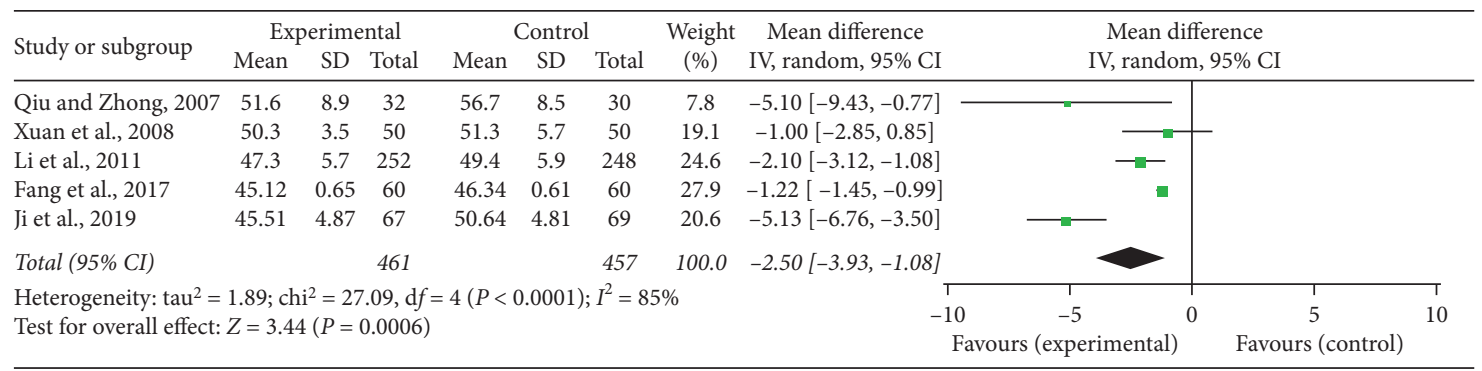

(b)

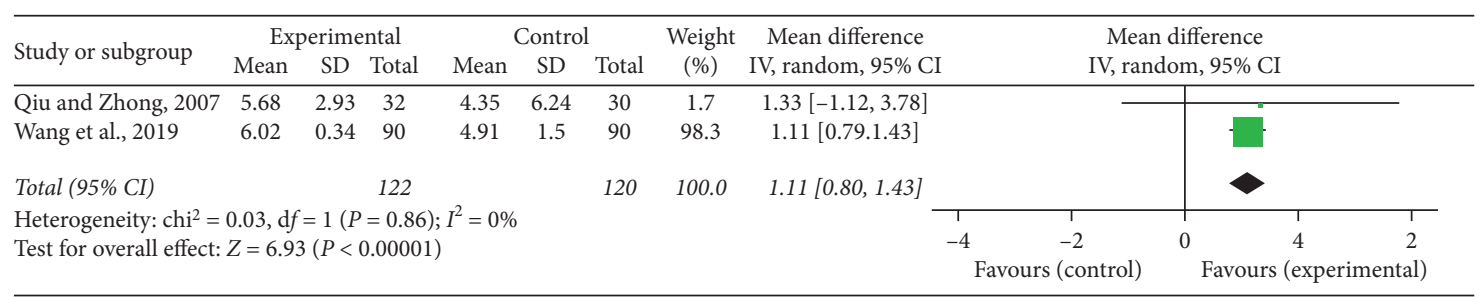

(c)

FIGURE 7: Forest plot of comparison in two groups for cardiac function indices. (a) Left ventricular ejection fraction; (b) left ventricular end diastolic diameter; (c) cardiac index. $I^{2}$ and $P$ are the criterion for the heterogeneity test, $\diamond$ pooled mean difference, $-\mathbf{\square}-$ mean difference, and $95 \% \mathrm{CI}$.

that CDDP could significantly decrease Hs-CRP for CHD patients. There were three $[32,33,38]$, five $[30,32,33,35,38]$, and three $[30,32,35]$ studies that reported TNF- $\alpha$, IL-6, and IL-8. Significant heterogeneity was respectively, found, among individual researches $\left(P<0.00001, \quad I^{2}=94 \% ; \quad P<0.00001, \quad I^{2}=88 \% ; \quad P=0.02\right.$, $\left.I^{2}=75 \%\right)$, and a random-effect model was adopted to carry out the meta-analysis. The pooled results showed that CDDP combined with PCI treatment significantly improved TNF- $\alpha$ $(\mathrm{MD}=-4.35, \quad 95 \% \quad \mathrm{CI} \quad(-5.99, \quad-2.71), \quad P<0.00001$; Figure $8(\mathrm{~b}))$, IL-6 $(\mathrm{MD}=-6.76$, 95\% CI $(-7.84,-5.68)$, $P<0.00001$; Figure $8(\mathrm{c}))$, and IL-8 $(\mathrm{MD}=-1.87,95 \% \mathrm{CI}$ $(-2.09,-1.66), P<0.00001$; Figure $8(d))$.

3.9. Adverse Reaction. Two $[21,28]$ of the included researches reported that no obvious adverse reaction occurred during treatment, and two $[33,34]$ recorded the incidence of adverse reactions. The adverse reactions consisted of gastrointestinal intolerance, dizziness, phlebitis, and pruritus. No heterogeneity $\left(P=0.89, I^{2}=0 \%\right)$ was found among individual studies, and a fixed-effect model was used to perform the meta-analysis. The pooled RR with 95\% CI showed that there was no difference about the incidence of adverse reactions between the experimental and control groups $(\mathrm{RR}=1.13,95 \% \mathrm{CI}(0.45,2.81), P=0.8$; Figure 9$)$.

3.10. Publication Bias. Funnel plot was employed to evaluate the publication bias. The publication bias was checked for MACE. As shown in Figure 10, the plots were basically symmetric, indicating that there was no obvious publication bias.

\section{Discussion}

4.1. Overview. Cardiovascular disease (CVD) is induced by more and more risk factors including improvement of people's living standards, changes in people's living habits, aging of population, and the constantly changing environment $[41,42]$. The morbidity and mortality of CVD remain high, and the burden of prevention and treatment of CVD is 


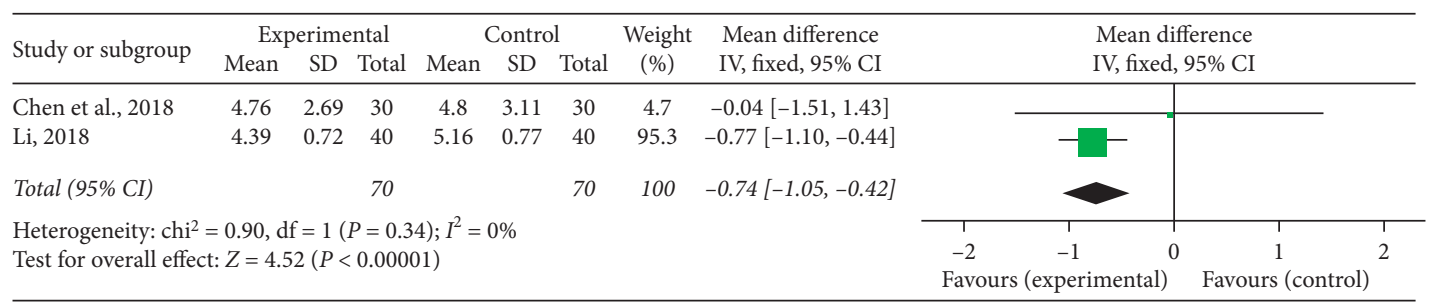

(a)

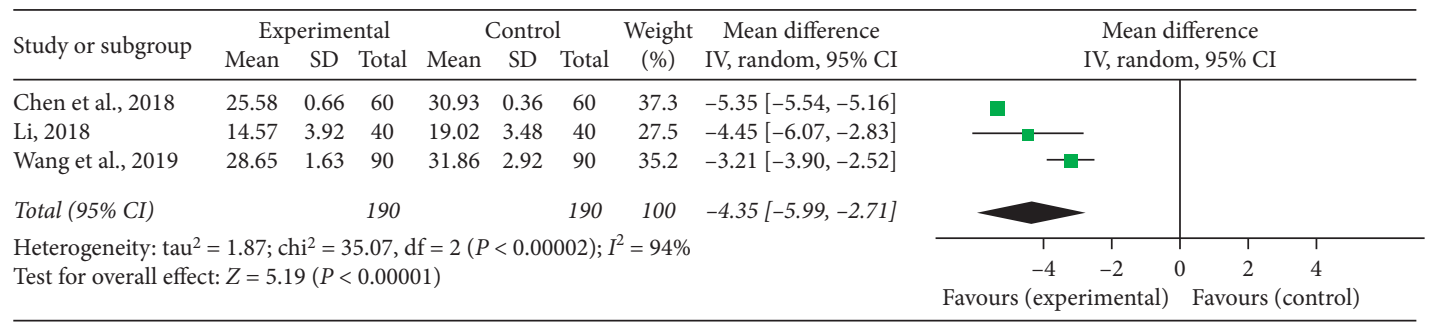

(b)

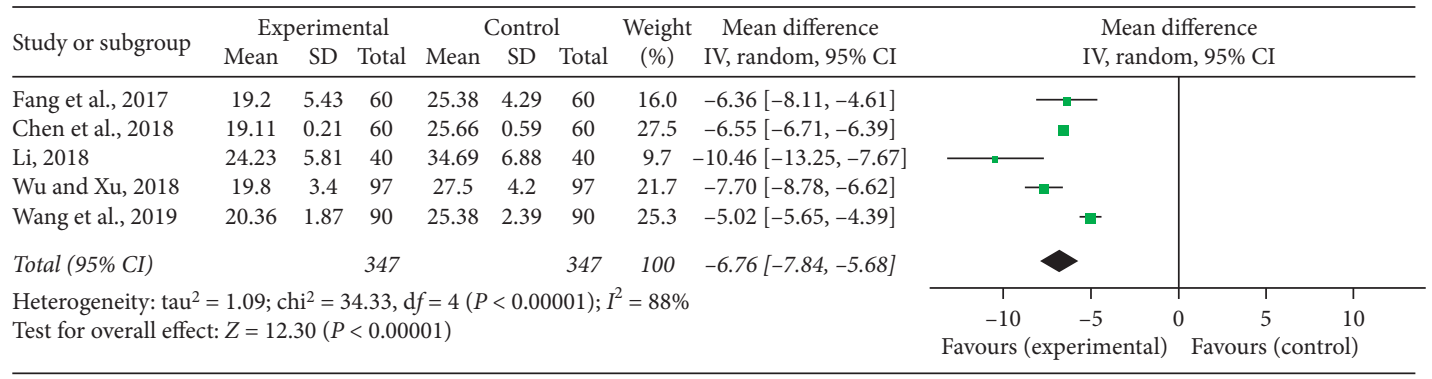

(c)

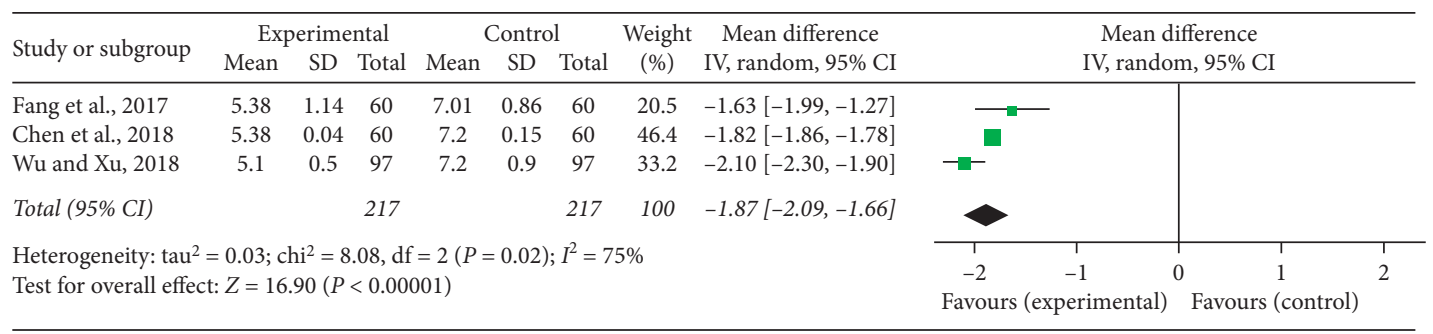

(d)

FIgURE 8: Forest plot of comparison in two groups for inflammatory indices. (a) High-sensitivity C-reactive protein; (b) tumor necrosis factor-alpha; (c) interleukin-6; (d) interleukin-8. $I^{2}$ and $P$ are the criterion for the heterogeneity test, $\downarrow$ pooled mean difference, $-\square-$ mean difference, and $95 \% \mathrm{CI}$.

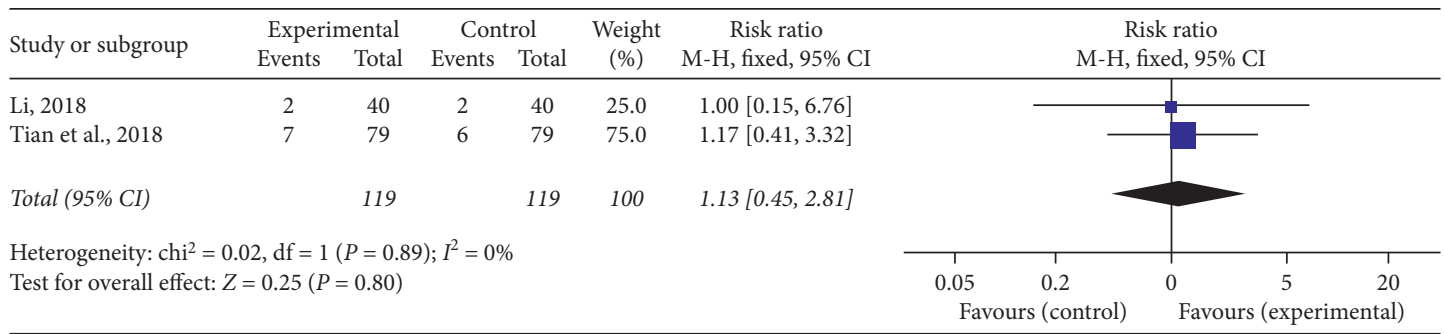

FIgURE 9: Forest plot of adverse reactions of CDDP plus PCI treatment compared to PCI treatment alone for CHD patients. $I^{2}$ and $P$ are the criterion for the heterogeneity test, $\bullet$ pooled risk ratio, - - risk ratio, and 95\% CI.

increasing [43]. It has become an important public health issue for human health. CHD is one of the most common and harmful CVD, characterized by high disability rate, mortality rate, and many complications, and seriously threatens public health [44]. Therefore, the researches on the treatment of CHD are of great significance to human health. 

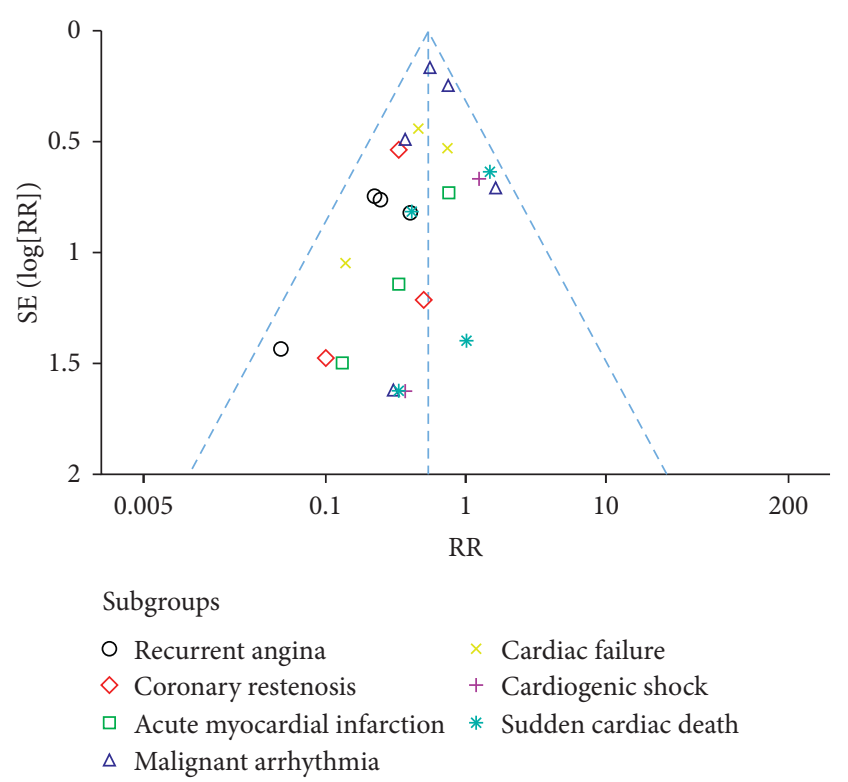

Figure 10: Funnel plot for the publication bias of major adverse cardiac events.

At present, PCI has become one of the main means for treating CHD because of its good therapeutic effect [45]. However, there has been a high incidence of adverse cardiovascular events in CHD patients after PCI treatment [46]. Given the circumstances, more effective and safe treatment is urgently needed for CHD patients in China and even the world.

Chinese clinicians have been looking for better treatments for $\mathrm{CHD}$ over the years. Traditional Chinese medicine (TCM) has been used to treat CHD for more than two thousand years. The therapeutic effect of Chinese traditional medicines for treating $\mathrm{CHD}$ is not bad and even stronger than some western medicines, and Chinese traditional medicines are characterized by little toxicity and side effect. Therefore, the application prospect of Chinese traditional medicines in CHD is great [47]. Along with the development of modern pharmaceutical technologies, oral preparations and injections for the prevention and treatment of CHD based on classical TCM prescriptions or theories have sprung up in large numbers [48].

CDDP is an excellent Chinese patent medicine developed by modern pharmaceutical technology based on the basic theory of TCM. Compared with the original tablet, CDDP has many advantages including small dosage, outstanding therapeutic effect, fewer side effects, and reduced gastrointestinal irritation [49]. Therefore, it is a commonly used Chinese medicine preparation in clinical practice in China. At present, Phase III clinical trial of CDDP has been completed in the United States. Then Tianjin Tasly Pharmaceutical Co., Ltd., will submit a new drug application to Food and Drug Administration [50]. CDDP is prepared from Salviae miltiorrhizae, Panax notoginseng, and Borneolum, and its major active constituents are tanshinol, protocatechuic aldehyde, salvianolic acid B, notoginsenoside, and so on [51]. Modern pharmacological studies have shown that these components are related to some effects, such as regulating lipid metabolism, improving vascular function, and inhibiting thrombosis [52]. CDDP have been widely used in the treatment of various CVD for many years [53]. However, there is lack of a comprehensive and systematic evaluation of CDDP for the treatment of CHD after PCI according to general international standards. Therefore, this study is aimed at providing an internationally recognized systematic assessment of the efficacy and safety of CDDP for treating CHD patients after PCI treatment.

This meta-analysis for the first time systematically assessed the clinical effect and safety of CDDP for treating CHD patients after PCI treatment. The incidence of MACE was used to evaluate the efficacy of CDDP combined with PCI treatment for CHD patients. Compared with PCI treatment alone, CDDP combined with PCI treatment was associated with remarkably lower MACE $(P<0.00001)$. Hemorheology indices, including WBV, PV, HCT, EAI, and FIB, were used to study the fluidity and deformability of blood in CHD participants. Compared with PCI treatment alone, CDDP combined with PCI treatment was associated with significantly lower WBV, PV, and EAI $(P<0.01)$. It indicated that CDDP contributed to improving the antithrombotic and anticoagulation effects. ET, FMD, and $\mathrm{NO}$ were used to evaluate vascular endothelial function in CHD patients. Our analysis results showed that, compared with PCI treatment alone, CDDP combined with PCI treatment was associated with significantly lower ET and higher FMD and NO $(P<0.01)$. Moreover, TC, TG, HDL-C, and LDL-C were used to assess the blood lipid in CHD patients. Results demonstrated that CDDP combined with PCI treatment significantly decreased the levels of TC and LDL-C in comparison with PCI treatment alone $(P<0.01)$. LVEF, LVEDD, and CI were used to estimate cardiac function in CHD patients. Compared with PCI treatment alone, CDDP combined with PCI treatment was associated with significantly lower LVEDD and higher LVEF and CI $(P<0.001)$. In addition, Hs-CRP, TNF- $\alpha$, IL-6, and IL-8 were applied to evaluate inflammation state in CHD patients after PCI. Results suggested that, compared with PCI treatment alone, CDDP combined with PCI treatment was associated with significantly lower Hs-CRP, TNF- $\alpha$, IL-6, and IL-8 $(P<0.00001)$. However, there was no difference about adverse reactions between the experimental and control groups $(P=0.8)$. It could be only temporarily concluded that CDDP is relatively safe without increasing the incidence of adverse reactions before including more eligible studies.

4.2. Limitations. Although comprehensive search and strict methodologies were employed to screen researches and investigate the therapeutic effect and safety associated with CDDP treatment, several potential limitations still existed in this meta-analysis that should be considered. Firstly, although an overall retrieval strategy was adopted to reduce the publication bias as far as possible, there was still a certain degree of selective bias that our meta-analysis only searched the Chinese and English databases and no reference was 
made to researches published in other languages. Secondly, all the eligible trials were conducted in China and most participants were Chinese. However, it is necessary to include some diverse population samples into the study to achieve more abundant and reliable results. Thirdly, the methodological quality in most of the eligible researches showed to be poor. Eleven of the 20 trials only referred to "randomization" but did not point out the specific random method. And all the included researches did not report allocation concealment and blinding method. Fourthly, we did not contact the authors using phone call or e-mail for more details of the included trials. Fifthly, there was statistically significant heterogeneity detected in several outcomes, such as FIB, ET, FMD, and NO. It is relatively difficult to study the heterogeneity in the outcomes of continuous variables. We were unable to perform a subgroup analysis for the small number of researches providing these outcomes and also failed to detect the sources of the heterogeneity after performing sensitivity analysis. It can be concluded that the heterogeneity came from two or more factors, such as gender, age, and duration of treatment. Finally, drug safety is significant to develop alternative medicines for health care. However, only two of the included researches reported adverse reactions.

4.3. Direction for the Future. According to our study, CDDP combined with PCI treatment is more effective for CHD patients compared with single PCI treatment. Therefore, this combination therapy regimen is recommended for widespread clinical use. Meanwhile, in consideration of the limitations existing in this meta-analysis, high-quality and large-scale RCTs, with good experimental design and methodological quality, are needed to investigate the clinical effect and safety of CDDP for CHD in the future.

\section{Conclusion}

The results showed that CDDP combined with PCI treatment remarkably reduced the incidence of MACE in CHD patients. Meanwhile, this combination improved blood rheology, vascular endothelial function, and cardiac function, decreased blood lipid, and exhibited anti-inflammatory effects. However, our findings must be interpreted with care for the limitations existing in this meta-analysis. Other rigorous and large-scale RCTs are in need to confirm these results.

$\begin{array}{ll}\text { Abbreviations } \\ \text { CDDP: } & \text { Compound Danshen dripping pill } \\ \text { CHD: } & \text { Coronary heart disease } \\ \text { CI: } & \text { Cardiac index } \\ \text { CVD: } & \text { Cardiovascular disease } \\ \text { EAI: } & \text { Erythrocyte aggregation index } \\ \text { ET: } & \text { Endothelin } \\ \text { FIB: } & \text { Fibrinogen } \\ \text { FMD: } & \text { Flow mediated dilation } \\ \text { HCT: } & \text { Hematocrit } \\ \text { HDL-C: } & \text { High density lipoprotein cholesterol }\end{array}$

Hs-CRP: High-sensitivity C-reactive protein

IL-6: Interleukin-6

IL-8: Interleukin-8

LDL-C: Low density lipoprotein cholesterol

LVEDD: Left ventricular end diastolic diameter

LVEF: Left ventricular ejection fraction

MACE: Major adverse cardiac events

NO: $\quad$ Nitric oxide

PCI: Percutaneous coronary intervention

PV: $\quad$ Plasma viscosity

RCTs: Randomized controlled trials

TC: $\quad$ Total cholesterol

TCM: Traditional Chinese medicine

TG: $\quad$ Triglyceride

TNF- $\alpha$ : Tumor necrosis factor-alpha

WBV: Whole blood viscosity.

\section{Data Availability}

The data used to support the findings of this study are included within the article.

\section{Conflicts of Interest}

All authors declared that there are no conflicts of interest.

\section{Authors' Contributions}

Cailan Li, Jianhui Xie, and Xiaobo Yang conceived and designed the experiments. Cailan $\mathrm{Li}$, Qian Li, Jiamin $\mathrm{Xu}$, Wenzhen $\mathrm{Wu}$, and Yuling $\mathrm{Wu}$ conducted the experiments. Cailan $\mathrm{Li}$, Qian $\mathrm{Li}$, and Jiamin $\mathrm{Xu}$ analyzed the data. Jianhui Xie and Xiaobo Yang contributed reagents/materials/analysis tools. Cailan Li, Qian Li, and Jiamin Xu wrote the paper. Jianhui Xie and Xiaobo Yang revised the paper.

\section{Acknowledgments}

This work was financially supported by the National Natural Science Foundation of China (Nos. 81673845 and 82003771), Science and Technology Planning Project of Guangdong Province (Nos. 2016A020226036 and 2017B030314166), Special Project of State Key Laboratory of Dampness Syndrome of Chinese Medicine (No. SZ2020ZZ03), Provincial Natural Science Foundation of Guangdong (No. 2019A1515010638), Science and Technology Research Project of Guangdong Provincial Hospital of Chinese Medicine (No. YN2018ZD02), and Guizhou Science and Technology Foundation of China (No. QKHPTRC[2018] 5772-021).

\section{References}

[1] A. Bechthold, H. Boeing, C. Schwedhelm et al., "Food groups and risk of coronary heart disease, stroke and heart failure: a systematic review and dose-response meta-analysis of prospective studies," Critical Reviews in Food Science and Nutrition, vol. 59, no. 7, pp. 1071-1090, 2019. 
[2] M. J. Pencina, A. M. Navar, D. Wojdyla et al., "Quantifying importance of major risk factors for coronary heart disease," Circulation, vol. 139, no. 13, pp. 1603-1611, 2019.

[3] A. M. Navar, "The evolving story of triglycerides and coronary heart disease risk," Journal of the American Medical Association, vol. 321, no. 4, pp. 347-349, 2019.

[4] M. De Hert, J. Detraux, and D. Vancampfort, "The intriguing relationship between coronary heart disease and mental disorders," Dialogues in Clinical Neuroscience, vol. 20, no. 1, pp. 31-40, 2018.

[5] S. Kachur, V. Chongthammakun, C. J. Lavie et al., "Impact of cardiac rehabilitation and exercise training programs in coronary heart disease," Progress in Cardiovascular Diseases, vol. 60, no. 1, pp. 103-114, 2017.

[6] F. Sanchis-Gomar, C. Perez-Quilis, R. Leischik, and A. Lucia, "Epidemiology of coronary heart disease and acute coronary syndrome," Annals of Translational Medicine, vol. 4, no. 13, pp. 1-12, 2016.

[7] P. H. Wirtz and R. Von Kanel, "Psychological stress, inflammation, and coronary heart disease," Current Cardiology Reports, vol. 19, no. 11, p. 111, 2017.

[8] N. V. K. Pothineni, S. Subramany, K. Kuriakose et al., "Infections, atherosclerosis, and coronary heart disease," European Heart Journal, vol. 38, no. 43, pp. 3195-3201, 2017.

[9] G. Veronesi, H. Tunstall-Pedoe, M. M. Ferrario et al., "Combined effect of educational status and cardiovascular risk factors on the incidence of coronary heart disease and stroke in European cohorts: implications for prevention," European Journal of Preventive Cardiology, vol. 24, no. 4, pp. 437-445, 2017.

[10] S. F. Su, M. Y. Chang, and C. P. He, "Social support, unstable angina, and stroke as predictors of depression in patients with coronary heart disease," Journal of Cardiovascular Nursing, vol. 33, no. 2, pp. 179-186, 2018.

[11] X. Y. Li, "Recommendations on the clinical use of compound danshen dripping pills," Chinese Medical Journal, vol. 130, no. 8, pp. 972-978, 2017.

[12] Y. F. Tian, L. Y. Li, R. Wang, and L. X. Ji, "A survey of cardioprotective effects of compound danshen dripping pills," Acta Chinese Medicine and Pharmacology, vol. 33, pp. 60-61, 2005.

[13] Q. Li, "Research progress and clinical application of compound danshen dripping pills," China Journal of Traditional Chinese Medicine and Pharmacy, vol. 33, no. 7, pp. 2989-2991, 2018.

[14] L. Xiao, "Research progress in pharmacological action and clinical application of compound danshen dripping pills," World Chinese Medicine, vol. 10, no. 7, pp. 1117-1123, 2015.

[15] X. Huang, G. J. Kou, and B. H. Wang, "The clinical research progress of compound danshen dripping pill," Lishizhen Medicine and Materia Medica Research, vol. 27, no. 5, pp. 1187-1190, 2016.

[16] D. Moher, A. Liberati, J. Tetzlaff, and D. G. Altman, "Preferred reporting items for systematic reviews and meta-analyses: the prisma statement," Annals of Internal Medicine, vol. 151, pp. 332-336, 2009.

[17] E. Braunwald, E. M. Antman, J. W. Beasley et al., "ACC/AHA guidelines for the management of patients with unstable angina and non-st-segment elevation myocardial infarction," Journal of the American College of Cardiology, vol. 36, no. 3, pp. 970-1062, 2000.

[18] D. Y. Li and L. K. Ma, "Progress in diagnosis and treatment of acute coronary syndrome," International Journal of Geriatric Psychiatry, vol. 28, pp. 173-177, 2007.
[19] H. Wang, F. Wang, Y. Z. Lin, J. Lu, G. M. Xu, and L. Liu, "Clinical observation of trimetazidine and compound Danshen dripping pills on percutaneous coronary intervention of coronary heart disease," Shaanxi Medical Journal, vol. 32, no. 3, pp. 252-254, 2003.

[20] Y. Q. Qiu and Y. M. Zhong, "Effects of compound danshen dripping pills on elderly patients with acute myocardial infarction undergoing emergent interventional therapy of coronary artery," Evaluation and Analysis of Drug-Use in Hospitals of China, vol. 7, no. 5, pp. 376-377, 2007.

[21] Y. J. Chen, Therapeutic Effect of Compound Danshen Dripping Pills on Coronary Heart Disease after PCI, pp. 14-20, Guangzhou University of Chinese Medicine, Guangzhou, China, 2008.

[22] C. H. Xuan, H. B. Sun, and D. Z. Ding, "Effect of compound danshen dripping pills on myocardial microcirculation of coronary heart disease patients after percutaneous coronary intervention," Lishizhen Medicine and Materia Medica Research, vol. 19, no. 11, pp. 2797-2798, 2008.

[23] R. Z. Zhao, Q. C. Fan, and B. Shi, "Effect of compound danshen dripping pills on hemorheology after PCI in patients with coronary heart disease," Chinese Journal of New Drugs, vol. 19, no. 2, pp. 130-132, 2010.

[24] G. P. Li, X. T. Zheng, H. Z. Wang et al., "Clinical effect of compound danshen dripping pills on percutaneous coronary intervention of acute ST-segment elevation myocardial infarction," Chinese Journal of Interventional Cardiology, vol. 19, no. 1, pp. 24-28, 2011.

[25] C. M. Tian, S. X. Li, and Y. X. Lv, "Therapeutic effect of compound danshen dripping pills and trimetazidine on coronary restenosis in patients with myocardial infarction after percutaneous coronary intervention," Chinese Community Doctors, vol. 13, no. 9, p. 168, 2011.

[26] X. P. Xia, "Clinical observation of compound danshen dripping pills on coronary restenosis in patients with coronary heart disease after percutaneous coronary intervention,", Journal of Today Health, vol. 13, no. 4, pp. 125-126, 2014.

[27] Y. Tang and H. Zhang, "Clinical research of compound danshen dripping pills in preventing and curing coronary heart disease with restenosis after percutaneous coronary intervention," Journal of Community Medicine, vol. 13, no. 21, pp. 16-19, 2015.

[28] Y. L. Yao, B. Yang, H. Kang, Y. M. Zhang, and Z. Y. He, "Effect of compound danshen dripping pills on hemorheology in acute coronary syndrome patients before and after percutaneous coronary intervention," Chinese Journal of Multiple Organ Diseases in The Elderly, vol. 14, no. 9, pp. 678-682, 2015.

[29] M. H. Li and J. Wang, "Effect of compound danshen dripping pills on hemorheology in patients with coronary heart disease after PCI," Chinese Medicine Modern Distance Education of China, vol. 14, no. 4, pp. 61-62, 2016.

[30] F. Fang, S. Y. Gan, G. R. Feng, H. Yu, and X. N. Zhang, "Effects of long-term use of compound danshen dripping pills on left ventricular remodeling and inflammatory factors in elderly patients with acute myocardial infarction after PCI," Progress in Modern Biomedicine, vol. 17, no. 3, pp. 544-546, 2017.

[31] Y. Y. Zhang, Influence of Compound Danshen Dripping Pills on PCI Efficacy in Patients with Acute ST-Segment Elevation Myocardial Infarction, pp. 3-8, Shanxi Medical University, Shanxi, China, 2017.

[32] H. Chen, D. M. Wei, L. Yu, G. Yin, J. S. Li, and X. N. Yang, "Effect of compound danshen dripping pills on serum inflammatory factors and $\mathrm{T}$ cell subset in elderly patients with 
acute myocardial infarction after PCI," Chinese Journal of Gerontology, vol. 38, no. 11, pp. 2586-2588, 2018.

[33] D. F. Li, "Clinical effect of compound danshen dripping pills and atorvastatin on improving inflammatory response and myocardial perfusion in patients with acute myocardial infarction after PCI," Prevention and Treatment of Cardio-Cerebral-Vascular Disease, vol. 18, no. 6, pp. 500-502, 2018.

[34] L. G. Tian, L. X. Zhang, C. Ji, Z. Y. Yuan, and J. Y. Liu, "Effects of alprostadil and compound danshen dripping pills on vascular endothelial function and cardiovascular adverse events in patients with coronary heart disease after PCI," Chinese Journal of Integrative Medicine on Cardio-Cerebrovascuiar Disease, vol. 16, no. 24, pp. 3680-3682, 2018.

[35] N. T. Wu and L. Q. Xu, "Effect of compound danshen dripping pills and statins on contrast-induced nephropathy and vascular function in patients with coronary heart disease after PCI," Journal of Hunan University of Chinese Medicine, vol. 38, no. 3, pp. 335-338, 2018.

[36] Y. Q. Ji, Z. X. Li, and J. M. Zuo, "Clinical observation of compound danshen dripping pills in the treatment of acute myocardial infarction after percutaneous coronary intervention," Journal of Critical Care in Internal Medicine, vol. 25, no. 3, pp. 243-245, 2019.

[37] X. S. Su, "Effects of compound danshen dripping pills on brain natriuretic peptide level and ventricular remodeling after percutaneous coronary intervention in patients with acute myocardial infarction," Chinese Journal of Practical Medicine, vol. 46, no. 12, pp. 110-114, 2019.

[38] R. T. Wang, M. X. Wu, and K. Y. Wang, "Effect of compound danshen dripping pills on expression of miR-1 in serum after percutaneous coronary intervention in patients with acute myocardial infarction," Chinese Journal of Integrated Traditional and Western Medicine in Intensive and Critical Care, vol. 26, pp. 302-305, 2019.

[39] A. D. Furlan, A. Malmivaara, and R. Chou, "Updated method guideline for systematic reviews in the cochrane back and neck group," Spine, vol. 40, no. 21, pp. 1660-1673, 2015.

[40] J. P. T. Higgins and S. G. Thompson, "Quantifying heterogeneity in a meta-analysis," Statistics in Medicine, vol. 21, no. 11, pp. 1539-1558, 2002.

[41] M. Kivimäki and A. Steptoe, "Effects of stress on the development and progression of cardiovascular disease," Nature Reviews Cardiology, vol. 15, no. 4, pp. 215-229, 2018.

[42] X. Ma, Y. X. Yang, N. A. Chen et al., "Meta-analysis for clinical evaluation of xingnaojing injection for the treatment of cerebral infarction," Frontiers in Pharmacology, vol. 8, pp. 1-12, 2017.

[43] C. J. Lagranha, T. L. A. Silva, S. C. A. Silva et al., "Protective effects of estrogen against cardiovascular disease mediated via oxidative stress in the brain," Life Sciences, vol. 192, pp. 190-198, 2018.

[44] S. S. Khan, H. Ning, J. T. Wilkins et al., "Association of body mass index with lifetime risk of cardiovascular disease and compression of morbidity," Journal of the American Medical Association Cardiology, vol. 3, no. 4, pp. 280-287, 2018.

[45] O. Kahkonen, P. Kankkunen, H. Miettinen, M. L. Lamidi, and T. Saaranen, "Perceived social support following percutaneous coronary intervention is a crucial factor in patients with coronary heart disease," Journal of Clinical Nursing, vol. 26, no. 9, pp. 1264-1280, 2019.

[46] S. Kodera, H. Morita, A. Kiyosue, J. Ando, and I. Komuro, "Cost-effectiveness of percutaneous coronary intervention compared with medical therapy for ischemic heart disease in
Japan," Circulation Journal, vol. 83, no. 7, pp. 1498-1505, 2019.

[47] K. J. Zhang, Q. Zheng, P. C. Zhu et al., "Traditional Chinese medicine for coronary heart disease: clinical evidence and possible mechanisms," Frontiers in Pharmacology, vol. 10, pp. 1-22, 2019.

[48] X. Yang, T. He, S. Han et al., "The role of traditional Chinese medicine in the regulation of oxidative stress in treating coronary heart disease," Oxidative Medicine and Cellular Longevity, vol. 2019, p. 13, 2019.

[49] X. G. Hu, "The difference between compound danshen dripping pills and compound danshen tablets," Chinese Community Doctors, vol. 36, no. 12, p. 116, 2020.

[50] Y. L. Xu, "Tasly compound danshen dripping pill has completed phase III clinical trial of US Food and drug administration," Tianjin Journal of Traditional Chinese Medicine, vol. 34, no. 2, pp. 74-75, 2017.

[51] J. Zhang, "Determination of six components in compound danshen dripping pills by HPLC-UV-ELSD," Herald of Medicine, vol. 33, no. 10, pp. 1375-1379, 2014.

[52] Y. Zou, "Advances in pharmacological effects and metabolism of compound danshen dripping pills," Chinese Journal of Clinical Rational Drug Use, vol. 11, no. 3, pp. 162-163, 2018.

[53] L. Y. Peng, "Pharmacological and clinical study of compound danshen dripping pills in cardiovascular diseases," China Health Industry, vol. 17, no. 19, pp. 186-188, 2013. 